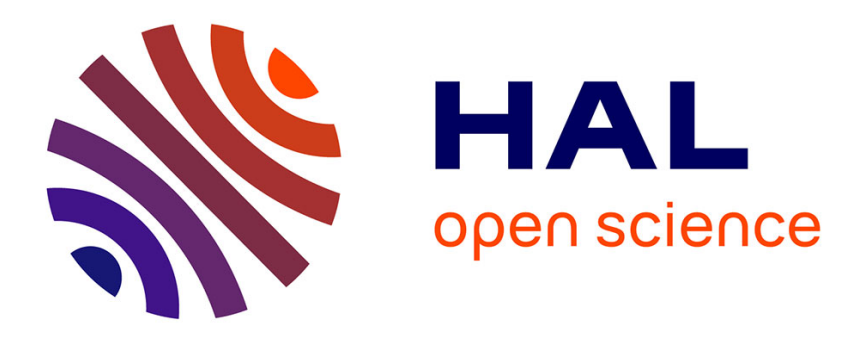

\title{
Études d'écoulements plans parallèles turbulents à parois mobiles
}

\author{
J.P. Chabrerie, G. Fournet, A. Mailfert
}

\section{To cite this version:}

J.P. Chabrerie, G. Fournet, A. Mailfert. Études d'écoulements plans parallèles turbulents à parois mobiles. Revue de Physique Appliquée, 1976, 11 (3), pp.353-368. 10.1051/rphysap:01976001103035300 . jpa-00244069

\section{HAL Id: jpa-00244069 https://hal.science/jpa-00244069}

Submitted on 1 Jan 1976

HAL is a multi-disciplinary open access archive for the deposit and dissemination of scientific research documents, whether they are published or not. The documents may come from teaching and research institutions in France or abroad, or from public or private research centers.
L'archive ouverte pluridisciplinaire HAL, est destinée au dépôt et à la diffusion de documents scientifiques de niveau recherche, publiés ou non, émanant des établissements d'enseignement et de recherche français ou étrangers, des laboratoires publics ou privés. 


\title{
REVUE DE PHYSIQUE APPLIQUÉE
}

\author{
Supplément au * Journal de Physique *
}

\author{
Classification \\ Physics Abstracts \\ $6.315-6.320-6.360$
}

\section{ÉTUDES D'ÉCOULEMENTS PLANS PARALLÈLES TURBULENTS A PAROIS MOBILES}

\author{
J. P. CHABRERIE, G. FOURNET et A. MAILFERT \\ Laboratoire de Génie Electrique de Paris $\left(^{*}\right)$ \\ Laboratoire Central des Industries Electriques \\ B. P. $n^{\circ} 8,92260$ Fontenay-aux-Roses \\ Institut National Polytechnique de Lorraine \\ Rue de la Citadelle 54-Nancy, France
}

(Reçu le 11 septembre 1975, accepté le 2 décembre 1975)

\begin{abstract}
Résumé. - La première partie de cet article est consacrée à l'étude des écoulements magnétohydrodynamiques plans parállèles, turbulents, de fluide newtonien incompressible, dans le cas où les parois limitant le canal de métal liquide sont mobiles l'une par rapport à l'autre dans le sens de l'écoulement. Une induction magnétique extérieure est appliquée perpendiculairement aux parois. La mise en mouvement du fluide est due à la fois à la mobilité des parois, supposées parfaitement lisses, et à la présence d'une source d'énergie électrique extérieure. Nous déterminons une expression analytique des dissipations en étudiant les variations de l'entropie de l'unité de masse du fluide en mouvement (en absence de phénomènes de thermoconduction). Une étude qualitative de la turbulence jointe à des considérations dimensionnelles (semblables à celles utilisées par Landau et Lifschitz dans le cas d'écoulements hydrodynamiques à parois fixes) nous a permis de proposer, d'une part, un critère de transition (laminaire-turbulent) pour ce type d'écoulement et, d'autre part, une loi régissant les dissipations.

La deuxième partie de cette étude décrit brièvement les solutions apportées aux principaux problèmes de M. H. D. posés pour l'utilisation des métaux liquides dans les machines homopolaires à inducteur supraconducteur.

La troisième partie permet de comparer les résultats expérimentaux aux résultats théoriques obtenus à l'aide d'une méthode de calcul basée sur l'étude décrite en première partie.

Abstract. - The first part of the paper is devoted to the study of turbulent hydromagnetic channel flows between two smooth planes of an incompressible conducting Newtonian liquid, where the boundary insulated planes are moving at two different speeds in the direction of the flow. An external magnetic field is applied normal to the walls. The flow is due to the mobility of the walls and to Laplace forces generated by an external power supply. By studying the total time derivative of the entropy of a unit mass of fluid moving in space, an analytical expression for the total magnetohydrodynamic losses is derived (thermal conduction phenomena are neglected). A qualitative study of turbulence and some dimensional considerations (similar to those used by Landau and Lifschitz in the case of hydrodynamic flows between fixed planes) lead us to propose a laminar-turbulent flow transition criterion and a law governing the dissipations.

The second part of this study describes briefly some solutions to the principal MHD problems encountered in homopolar superconducting electric machines when liquid metals are used.

The third part compares the experimental results with theoretical results obtained numerically, based on the study described in the first part of this paper.
\end{abstract}

Introduction. Pour réaliser des machines électriques possédant un couple volumique élevé, il est intéressant de mettre en œuvre des systèmes ne comportant pas de matériaux ferromagnétiques [1]. L'emploi de conducteurs conventionnels pour réaliser l'inducteur restreint ces machines à un usage impulsionnel [2]. Par contre l'utilisation de matériaux supraconducteurs dans des inducteurs de moteurs homopolaires permet d'effectuer

(*) Associé au C. N. R. S. en régime permanent une conversion électromécanique de puissance qui bénéficie simultanément d'un couple volumique élevé et d'un bon rendement [3], [4] et [5]. Dans cette classe de machines, l'obtention de ces caractéristiques est indissociablement liée à la réalisation de contacts électriques d'induit de haute qualité. Les métaux liquides sont une solution séduisante pour ce problème, mais leur mise en œuvre efficace nécessite la compréhension des phénomènes magnétohydrodynamiques qui prennent alors naissance. 
Le but du présent article est d'établir dans une première partie les lois d'écoulements magnétohydrodynamiques de métaux liquides dans certaines configurations particulières, puis dans une seconde partie d'appliquer ces résultats à l'étude du mode de contacts retenu pour la réalisation d'un moteur homopolaire dont nous indiquerons dans une troisième partie les principales caractéristiques.

1. Etude théorique des écoulements plans parallèles en régime turbulent, permanent en moyenne, en présence de parois mobiles l'une par rapport à l'autre (fluide incompressible). - 1.1 CAS GÉNÉRAL OU LA MISE EN MOUVEMENT DU FLUIDE EST DUE A LA FOIS AUX FORCES DE LAPLACE ET A UN GRADIENT DE PRESSION MOTRICE. Considérons un écoulement magnétohydrodynamique plan turbulent, à débit constant s'effectuant dans la direction Ox (Fig. 1a) entre deux plans parallèles lisses isolants (1) et (2) animés par rapport au référentiel fixe $\mathcal{R}(O x y z)$ de vitesse $\mathbf{U}_{1}$ et $\mathbf{U}_{2}$ suivant $\mathbf{O x}$ (avec

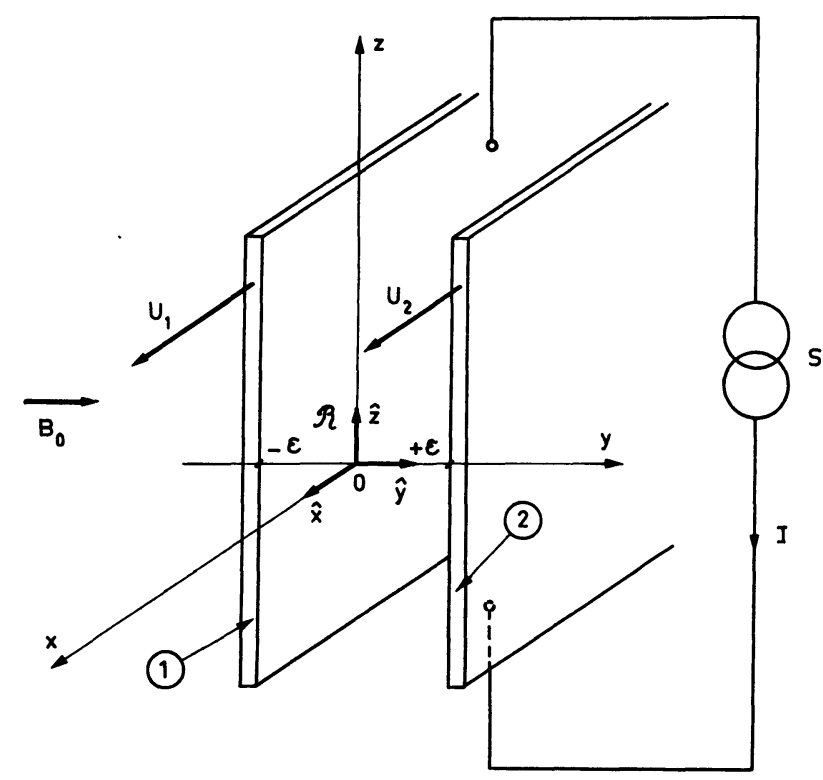

FIG. 1a. - Modèle d'écoulement plan-parallèle à parois mobiles.

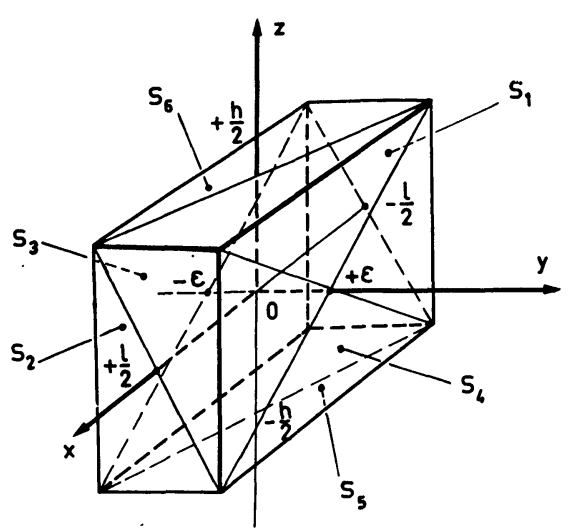

Fig. 1b. - Surface $S=\sum_{j=1}^{6} S_{j}$ limitant le volume de fluide dans lequel on calcule les dissipations.
$\left.\mathbf{U}_{1} \neq \mathbf{U}_{2}\right)$. Nous supposerons que le fluide est, de plus $\left({ }^{1}\right)$, soumis à une forme d'entraînement produite à la fois par un gradient de pression motrice suivant $x$ et par des forces de Laplace résultant de l'action de l'induction extérieure $\overrightarrow{\mathbf{B}}_{0}$ (appliquée suivant $\mathbf{O y}$ ) sur un courant moyen non nul traversant le modèle dans le sens Oz. On suppose en outre la présence en $z= \pm \infty$ de plans parfaitement conducteurs.

Notations adoptées : Nous désignerons par:

$$
\text { - }<g>(x, y, z)=\frac{1}{t_{0}} \int_{t_{1}}^{t_{1}+t_{0}} g(x, y, z, t) \mathrm{d} t,
$$

la moyenne au cours du temps de la grandeur instantanée $g$. On suppose que l'intervalle de temps $t_{0}$ est assez grand pour que la moyenne calculée $\langle g\rangle$ ne dépende plus ni de $t_{0}$ ni de $t_{1}$ (le régime étant permanent en moyenne).

$$
\text { - } g^{\prime}(x, y, z, t)=g(x, y, z, t)-<g>(x, y, z),
$$

la fluctuation temporelle à laquelle est soumise la grandeur $g$ (il vient immédiatement $\left\langle g^{\prime}\right\rangle=0$ ).

$$
\text { - } \bar{h}(x, z, t)=\frac{1}{2 \varepsilon} \int_{-\varepsilon}^{+\varepsilon} h(x, y, z, t) \mathrm{d} y,
$$

la valeur moyenne, sur l'épaisseur $2 \varepsilon$ de la veine liquide, de la grandeur $h$.

- $\mathbf{U}_{1}$ et $\mathbf{U}_{2}$, les vitesses constantes des parois (mesurées dans $\mathcal{R}$ ).

- $\rho, \eta, \gamma$, masse volumique, viscosité et conductivité électrique du fluide.

- $\mu_{0}$, perméabilité magnétique du vide.

- $p$, la valeur instantanée de la pression en un point $M(x, y, z)$.

- $e$, la demi-épaisseur de la veine liquide (voir Fig. 1a).

$\widetilde{\mathbf{B}}, \mathbf{j}, \mathbf{E}, \breve{\mathbf{H}}$ l'induction magnétique, la densité de courant et les champs électrique et magnétique en $M$ au temps $t$ (on supposera partout $\overleftrightarrow{\mathbf{B}}=\mu_{0} \breve{\mathbf{H}}$ ) (1)

$$
\left.\frac{\mathrm{d} I_{z}}{\mathrm{~d} x}=\mathrm{J}=2 \varepsilon<\bar{j}_{z}\right\rangle,
$$

la densité linéaire $\left(\mathrm{A} \cdot \mathrm{m}^{-1}\right)$ du courant injecté par la source extérieure $S$.

- $\mathrm{V}(x, y, z, t)$, la vitesse instantanée du fluide au point $M(x, y, z)$, de composante $v_{i}$ sur l'axe $\mathbf{O x}_{i}$ mesurée dans $\mathcal{R}$.

- $\pi$, le tenseur densité de flux d'impulsion dont la composante $\pi_{i k}(i, k=x, y, z)$ a pour expression [6] :

$\pi_{i k}=\rho v_{i} v_{k}+p \delta_{i k}-\sigma_{i k}-\mu_{0}\left(H_{i} H_{k}-\frac{1}{2} H^{2} \delta_{i k}\right)$,

$\delta_{i k}$ étant le symbole de Kronecker.

- Le premier terme de l'expression de $\pi_{i k}$ corres-

(1) Pour tenir compte du fait que la mobilité des parois peut constituer à elle seule une force d'entraînement. 
pond au transport d'impulsion lié au déplacement mécanique des différentes parties du fluide d'un lieu à un autre.

- Le second terme correspond aux forces de pression s'exerçant sur le fluide.

- Le troisième terme correspond au transport visqueux irréversible et a pour expression dans le cas envisagé d'un fluide incompressible :

$$
\sigma_{i k}=\eta\left(\frac{\partial v_{i}}{\partial \pi_{k}}+\frac{\partial v_{k}}{\partial \pi_{i}}\right)
$$

composante du tenseur $\boldsymbol{\sigma}$.

- Le dernier terme correspond aux forces magnétiques s'exerçant sur le fluide (tensions de Maxwell).

1.1.1 Equations générales. - Nous supposerons les distances suivant $\mathbf{O x}$ et $\mathbf{O z}$ suffisamment grandes pour pouvoir négliger les effets d'extrémité. Les conditions de symétrie du problème imposent aux moyennes dans le temps des grandeurs autres que la pression de ne dépendre que de $y$ soit :

et

$$
\left.\begin{array}{c}
\frac{\partial}{\partial \pi}<g>=\frac{\partial}{\partial z}<g>=0 \\
<p>=<p>(x, y)
\end{array}\right\} .
$$

La vitesse instantanée du fluide en un point $M(x, y, z)$ mesurée dans $\mathcal{R}$ s'écrit donc :

$$
\begin{aligned}
\mathbf{V}(x, y, z, t)=< & \mathbf{V}>(y)+\mathbf{V}^{\prime}(x, y, z, t)= \\
=\hat{x}[ & \left.<v_{x}>(y)+v_{x}^{\prime}(x, y, z, t)\right]+ \\
& +\hat{y} v_{y}^{\prime}(x, y, z, t)+\hat{z} v_{z}^{\prime}(x, y, z, t)
\end{aligned}
$$

$\hat{x}, \hat{y}, \hat{z}$ étant les vecteurs unitaires sur les axes correspondants.

L'équation générale de la magnétohydrodynamique des fluides visqueux incompressibles s'écrit ici [6]

$$
\frac{\partial}{\partial t}(\rho \mathbf{V})=\rho \frac{\partial \mathbf{V}}{\partial t}=-\operatorname{Div} \tilde{\pi}
$$

où la composante sur l'axe $\mathbf{O} \mathbf{x}_{i}$ de Div $\pi$ est définie par

$$
[\operatorname{Div} \tilde{\pi}]_{i}=\sum_{k} \frac{\partial}{\partial x_{k}}\left(\pi_{i k}\right)
$$

en tenant compte de l'incompressibilité (Div $\mathbf{V}=0$ ) la relation (6) devient ainsi

$$
\rho \frac{\partial \mathbf{V}}{\partial t}=-\rho \operatorname{Div}(\mathbf{V V})-\operatorname{grad} p+\eta \Delta \mathbf{V}+\mathbf{j} \wedge \overleftrightarrow{\mathbf{B}}
$$

La composante suivant $\mathbf{O} \mathbf{x}_{i}$ du flux d'impulsion relatif à un élément de surface orienté $(\mathrm{d} S=\mathbf{n} \mathrm{d} S)$ a pour expression :

$$
\mathrm{d} f_{i}=\sum_{k} \pi_{i k} n_{k} \mathrm{~d} S
$$

où $n_{x}, n_{y}, n_{z}$ sont les composantes, dans $\mathcal{R}$, du vecteur unitaire $\mathbf{n}$ normal à l'élément de surface $\mathrm{d} S$. La composante suivant $\mathbf{O x}$ du flux d'impulsion à travers l'unité d'aire perpendiculaire en $M(x, y, z)$ à Oy s'écrit donc d'après (9), et (2), pour une orientation de la normale $\mathbf{n}$ telle que $\mathbf{n}=-\hat{y}(\hat{y}$ : vecteur unitaire sur $\mathbf{O y})$

$$
T=-\pi_{x y}=-\rho v_{x} v_{y}+\sigma_{x y}+\mu_{0} H_{x} H_{y} .
$$

D'après le sens choisi pour $n, T$ correspond à la densité de flux d'impulsion cédée de manière continue des couches fluides situées en $y+0$ à celles situées en $y$ - 0 . La grandeur $T$ correspond donc ainsi à la contrainte exercée sur l'élément de surface $\mathrm{d} x \mathrm{~d} z$ par les couches situées en $y+0$, sur celles situées en $y-0$.

Compte tenu des éq. (3) et (5), la moyenne dans le temps de $\langle T\rangle$ a pour expression :

$$
\begin{aligned}
& \left.<T>=-\rho<v_{x}^{\prime} v_{y}^{\prime}\right\rangle+\eta\left\langle\frac{\partial v_{x}}{\partial y}\right\rangle+ \\
& \quad+\mu_{0}<H_{x}>\left\langle H_{y}>+\mu_{0}<H_{x}^{\prime} H_{y}^{\prime}\right\rangle .
\end{aligned}
$$

L'utilisation des relations (4) et $\operatorname{de} \operatorname{div} \mathbf{j}=\operatorname{div} \breve{\mathbf{B}}=0$ permet d'écrire $\mathbf{j}$ et $\overleftrightarrow{\mathbf{B}}$ sous la forme

$$
\begin{array}{ll}
j_{x}=<j_{x}>+j_{x}^{\prime} & B_{x}=<B_{x}>+B_{x}^{\prime} \\
j_{y}=j_{y}^{\prime} & B_{y}=B_{0}+B_{y}^{\prime} \\
j_{z}=\left\langle j_{z}>+j_{z}^{\prime}\right. & B_{z}=<B_{z}>+B_{z}^{\prime} .
\end{array}
$$

En projetant sur les axes $\mathbf{O x}, \mathbf{O y}, \mathbf{O z}$ l'éq. (6), compte tenu de (2) et (7) on obtient, en régime statistiquement stationnaire :

$$
\begin{aligned}
& O=-\frac{\partial}{\partial x}<\pi_{x x}>-\frac{\partial}{\partial y}<\pi_{x y}>=-\frac{\partial<p>}{\partial x}-\frac{\partial}{\partial y}\left(\rho<v_{x}^{\prime} v_{y}^{\prime}>-\eta \frac{\partial<v_{x}>}{\partial y}\right)+ \\
& +B_{0} \frac{\partial<H_{x}>}{\partial y}+\mu_{0} \frac{\partial}{\partial y}<H_{x}^{\prime} H_{y}^{\prime}> \\
& O=-\frac{\partial}{\partial y}<\pi_{y y}>=\frac{\partial<p>}{\partial y}-\rho \frac{\partial}{\partial y}\left\langle v_{y}^{\prime 2}\right\rangle+\mu_{0} \frac{\partial}{\partial y}\left(<H_{y}^{\prime 2}>-\frac{<H^{\prime 2}>}{2}\right)- \\
& -\frac{\mu_{0}}{2} \frac{\partial}{\partial y}\left(<H_{x}^{2}>+<H_{z}^{2}>-<H_{y}^{2}>\right) \\
& O=-\frac{\partial}{\partial y}\left\langle\pi_{z y}>=-\rho \frac{\partial}{\partial y}\left\langle v_{y}^{\prime} v_{z}^{\prime}\right\rangle+B_{0} \frac{\partial<H_{z}>}{\partial y}+\mu_{0} \frac{\partial}{\partial y}<H_{y}^{\prime} H_{z}^{\prime}\right\rangle .
\end{aligned}
$$


Dans le métal liquide la projection de la relation :

$$
\langle\mathbf{j}\rangle=\gamma\left(\langle\mathbf{E}\rangle+\langle\mathbf{V}\rangle \wedge\langle\overleftrightarrow{\mathbf{B}}\rangle+\left\langle\mathbf{V}^{\prime} \wedge \breve{B}^{\prime}\right\rangle\right)=\operatorname{rot}\langle\overleftrightarrow{\mathbf{H}}\rangle
$$

conduit à

$$
\begin{aligned}
& <J_{x}>=\gamma\left(<E_{x}>+<v_{y}^{\prime} B_{z}^{\prime}>-<v_{z}^{\prime} B_{y}^{\prime}>\right)=\frac{\left.\partial<H_{z}\right\rangle}{\partial y} \\
& <J_{y}>=\gamma\left(<E_{y}>-<v_{x}><B_{z}>+<v_{z}^{\prime} B_{x}^{\prime}>-<v_{x}^{\prime} B_{z}^{\prime}>\right)=0 \\
& <J_{z}>=\gamma\left(<E_{z}>+<v_{x}>B_{0}+<v_{x}^{\prime} B_{y}^{\prime}>-<v_{y}^{\prime} B_{x}^{\prime}>\right)=-\frac{\left.\partial<H_{x}\right\rangle}{\partial y}
\end{aligned}
$$

Nous appellerons $\left\langle T_{\mathrm{p}_{1}}\right\rangle$ et $\left\langle T_{\mathrm{p}_{2}}\right\rangle$ les valeurs des contraintes pariétales prises par $\langle T\rangle$ en $y=-\varepsilon$ et $+\varepsilon$ respectivement. D'après (10) et (11) il vient, en remarquant que sur ces parois $\mathbf{v}^{\prime}=0$,

$$
<T_{\mathrm{p}_{2}^{1}}>=\eta\left(\frac{\partial<v_{x}>}{\partial y}\right)_{y=\mp \varepsilon}+B_{0}\left(<H_{x}>\right)_{y=\mp \varepsilon}+\mu_{0}\left(<H_{x}^{\prime} H_{y}^{\prime}>\right)_{y=\mp \varepsilon}
$$

que nous écrirons pour alléger les notations

$$
<T_{p_{2}^{1}}>=<\tau_{p_{2}^{1}}>+B_{0}\left(<H_{x}>\right)_{y=\mp \varepsilon}+\mu_{0}\left(<H_{x}^{\prime} H_{y}^{\prime}>\right)_{y=\mp \varepsilon}
$$

avec

$$
<\tau_{\mathrm{p}_{2}^{1}}>=\eta\left(\frac{\partial<v_{x}>}{\partial y}\right)_{y=\mp \varepsilon} .
$$

D'autre part, les éq. (12), (13) et (14) montrent que grad $\langle p\rangle$ ne dépend que de $y$, par conséquent, la forme générale de $\langle p\rangle\left[\right.$ compte tenu de ce que $\left.\frac{\partial}{\partial x}\left(\frac{\partial\langle p\rangle}{\partial x}\right) \equiv 0\right]$ est :

$$
<p>(x, y)=\alpha x+f(y)
$$

où $\alpha=\partial\langle p\rangle / \partial x$ est une constante.

L'éq. (12) prend ainsi la forme

$$
0=-\alpha+\frac{\partial}{\partial y}<T>
$$

d'où

$$
<T>=<T_{\mathbf{p}_{1}}>+\alpha(y+\varepsilon)
$$

et

$$
<T_{\mathrm{p}_{2}}>=<T_{\mathrm{p}_{1}}>+2 \alpha \varepsilon .
$$

1.1.2 Calcul des dissipations magnétohydrodynamiques totales. - L'équation de conservation de l'énergie s'écrit en absence de thermoconduction, et dans le cas général d'un fluide compressible [6] :

$$
\frac{\partial}{\partial t}\left(\rho \frac{v^{2}}{2}+\rho \varepsilon+\frac{\breve{\mathbf{H}} \cdot \overleftrightarrow{\mathbf{B}}}{2}\right)=-\operatorname{div}\left[\rho \mathbf{V}\left(\frac{v^{2}}{2}+\omega\right)+\mathbf{E} \wedge \overleftrightarrow{\mathbf{H}}-\mathbf{V} . \tilde{\Gamma}\right]
$$

où $\delta$ et

$$
\omega=\varepsilon+\frac{p}{\rho}
$$

sont respectivement l'énergie interne et l'enthalpie par unité de masse du fluide, et $(\mathbf{V} . \Gamma)$ un vecteur dont la composante sur l'axe $\mathbf{O} \mathbf{x}_{i}$ est :

$$
(\mathbf{V} . \tilde{\Gamma})_{i}=\sum_{k} v_{k} \Gamma_{i k}
$$

avec

$$
\Gamma_{i k}=\sigma_{i k}+b \delta_{i k} \operatorname{div} \mathbf{V}
$$


et

$$
b=-\frac{2}{3} \eta+\zeta
$$

( $\eta$ et $\zeta$ étant les coefficients de viscosité).

Les relations (25) et (26) donnent :

$$
\frac{\partial}{\partial t}\left(\rho \frac{v^{2}}{2}+\frac{\breve{\mathbf{H}} \cdot \overleftrightarrow{\mathbf{B}}}{2}\right)+\operatorname{div}(\rho \mathbf{V} \boldsymbol{)})+\frac{\partial}{\partial t}(\rho \varepsilon)=-\operatorname{div}\left[\rho \mathbf{V}\left(\frac{v^{2}}{2}+\frac{p}{\rho}\right)+\mathbf{E} \wedge \breve{\mathbf{H}}-\mathbf{V} \cdot \tilde{\mathbf{\Gamma}}\right]
$$

En utilisant la relation thermodynamique valable pour des phénomènes réversibles :

$$
\frac{\mathrm{d} \delta}{\mathrm{d} t}=T \frac{\mathrm{d} S}{\mathrm{~d} t}+\frac{p}{\rho^{2}} \frac{\partial \rho}{\partial t}=\frac{\partial \mathcal{E}}{\partial t}+(\mathbf{V} \cdot \mathbf{g r a d}) \varepsilon
$$

(où $S$ désigne l'entropie par unité de masse du fluide) et l'équation de continuité :

$$
0=\frac{\partial p}{\partial t}+\operatorname{div} \rho \mathbf{V}=\frac{\partial p}{\partial t}+\mathbf{V} \cdot \operatorname{grad} \rho+\rho \operatorname{div} \mathbf{V}=\frac{\mathrm{d} \rho}{\mathrm{d} t}+\rho \operatorname{div} \mathbf{V}
$$

on obtient :

$$
\frac{\partial \mathcal{E}}{\partial t}+\mathbf{V} \cdot \operatorname{grad} \delta=T \frac{\mathrm{d} S}{\mathrm{~d} t}-\frac{p}{\rho} \operatorname{div} \mathbf{V}
$$

Par ailleurs grâce à (29) et ensuite (30), il vient

$$
\operatorname{div}(\rho \mathbf{V} \mathcal{E})+\frac{\partial}{\partial t}(\rho \mathcal{E})=\rho \mathbf{V} \cdot \operatorname{grad} \mathcal{E}+\rho \frac{\partial \mathcal{E}}{\partial t}=\rho T \frac{\mathrm{d} S}{\mathrm{~d} t}-p \operatorname{div} \mathbf{V}
$$

d'où en définitive $[\mathrm{cf} .(27)]$

$$
\begin{aligned}
\rho T \frac{\mathrm{d} S}{\mathrm{~d} t}= & -\operatorname{div}\left[\rho \mathbf{V}\left(\frac{v^{2}}{2}+\frac{p}{\rho}\right)+\mathbf{E} \wedge \overleftrightarrow{\mathbf{H}}-\mathbf{V} . \Gamma\right] \\
& -\frac{\partial}{\partial t}\left(\rho \frac{v^{2}}{2}+\frac{\breve{H} \cdot \breve{B}}{2}\right)+p \operatorname{div} \mathbf{V}
\end{aligned}
$$

$\frac{\mathrm{d} S}{\mathrm{~d} t}$ représente la variation de l'entropie de l'unité de masse du fluide en mouvement, $\rho T \frac{\mathrm{d} S}{\mathrm{~d} t}$ est donc la source d'entropie, c'est-à-dire, la puissance dissipée dans l'unité de volume du fluide et dans l'unité de temps.

En moyenne dans le temps, en considérant de nouveau le cas de l'écoulement turbulent, permanent en moyenne, d'un fluide incompressible, les dissipations à l'intérieur d'un volume $W$ de fluide seront données par l'expression :

$$
\left\langle P_{\mathrm{d}}\right\rangle=-\iint_{S(W)}\left[\left\langle\rho \mathbf{V}\left(\frac{v^{2}}{2}+\frac{p}{\rho}\right)\right\rangle+\langle\mathbf{E} \wedge \widetilde{\mathbf{H}}\rangle-\langle\mathbf{V} . \tilde{\boldsymbol{\sigma}}\rangle\right] \cdot \mathbf{n}_{\mathrm{S}} \mathrm{d} S=-\iint_{S(W)}\langle\mathbf{P}\rangle \cdot \mathbf{n}_{\mathrm{S}} \mathrm{d} S
$$

où $\mathbf{n}_{\mathbf{S}}$ désigne la normale sortante; le vecteur $\langle\mathbf{P}\rangle$ est la densité de flux de puissance dissipée. Nous allons appliquer ce résultat au cas de l'écoulement représenté sur la figure $1 a$. Le volume $W$ du fluide où sont calculées les dissipations est un parallélépipède rectangle, limité par les parois (1) et (2), d'une part et quatre plans, deux à deux perpendiculaires aux axes $\mathbf{O x}$ et $\mathbf{O z}$, d'autre part.

Nous désignerons par (cf. Fig. $1 b$ ) :

$-S_{1}, S_{2}$ les surfaces délimitant le volume $W$ et perpendiculaires à l'axe Ox respectivement en $x=-l / 2$ et $x=+l / 2$.

- $S_{3}, S_{4}$ les faces du parallélépipède correspondant aux parois solides (1) et (2) limitant la veine liquide respectivement en $y=-\varepsilon$ et $+\varepsilon$.

$-S_{5}, S_{6}$ les faces constituées par les portions de plans orthogonaux à l'axe $\mathbf{O z}$, respectivement en $z=-h / 2$ et $+h / 2$.

Les moyennes dans le temps n'étant fonction que de $y[$ cf. (4)], à l'exception de la pression $\langle p\rangle(x, y)$, on en déduit :

$$
\iint_{S_{1}+S_{2}}\left(\left\langle\rho \mathbf{V} \cdot \frac{v^{2}}{2}\right\rangle+\langle\mathbf{E} \wedge \overleftrightarrow{\mathbf{H}}\rangle-\langle\mathbf{V} \cdot \tilde{\sigma}\rangle\right) \cdot \mathrm{n}_{\mathrm{S}} \mathrm{d} S=0
$$


et

$$
\iint_{S_{5}+S_{6}}\left(\left\langle\rho \mathbf{V}\left(\frac{v^{2}}{2}+\frac{p}{\rho}\right)\right\rangle+\langle\mathbf{E} \wedge \breve{\mathbf{H}}\rangle-\langle\mathbf{V} . \tilde{\boldsymbol{\sigma}}\rangle\right) \cdot \mathbf{n}_{\mathrm{S}} \mathrm{d} S=0 .
$$

Les fluctuations de vitesse devant s'annuler sur les parois (1) et (2) il vient [cf. (5)] :

$$
\iint_{S_{3}+S_{4}}\left[\left\langle\rho v_{y}^{\prime}\left(\frac{v^{2}}{2}+\frac{p}{\rho}\right)\right\rangle-\left\langle\left(\mathbf{V}^{\prime} \cdot \tilde{\boldsymbol{\sigma}}\right)_{y}\right\rangle\right] \cdot \mathrm{n}_{\mathrm{S}} \mathrm{d} S=0 .
$$

Le flux de $<\mathbf{P}>$ à travers $S(W)$ [cf. (33)] se réduit donc à l'expression :

$$
\begin{aligned}
\iint_{S(W)}<P>\cdot \mathbf{n}_{\mathrm{S}} \mathrm{d} S=\left\langle\left[\iint_{S_{1}}(\right.\right. & \left.v_{x}>+v_{x}^{\prime}\right)\left(<p>+p^{\prime}\right)(-\mathrm{d} y \mathrm{~d} z)+ \\
& +\iint_{S_{2}}\left(<v_{x}>+v_{x}^{\prime}\right)\left(<p>+p^{\prime}\right) \mathrm{d} y \mathrm{~d} z+\iint_{S_{3}}(\mathbf{E} \wedge \breve{\mathbf{H}})_{y}(-\mathrm{d} x \mathrm{~d} z) \\
& \left.\left.+\iint_{S_{4}}(\mathbf{E} \wedge \breve{\mathbf{H}})_{y} \mathrm{~d} x \mathrm{~d} z\right]\right\rangle+\iint_{S_{3}}(<\mathbf{V}>\cdot<\tilde{\boldsymbol{\sigma}}>)_{y} \mathrm{~d} x \mathrm{~d} z \\
& +\iint_{S_{4}}(<\mathbf{V}>\cdot<\tilde{\boldsymbol{\sigma}}>)_{y}(-\mathrm{d} x \mathrm{~d} z)
\end{aligned}
$$

Nous utiliserons pour simplifier, les notations suivantes :

$$
\iint_{S(W)}<\mathrm{P}>\cdot \mathrm{n}_{\mathrm{S}} \mathrm{d} S=\langle\mathrm{I}>+<\mathrm{II}>+<\mathrm{III}>+<\mathrm{IV}>+<\mathrm{V}>+<\mathrm{VI}\rangle
$$

expression dans laquelle les chiffres romains utilisés, représentent les intégrales doubles de l'éq. (34) dans l'ordre suivant lequel elles apparaissent dạns le membre de droite.

a) Compte tenu de l'éq. (21) et de la relation :

$$
\frac{\partial}{\partial x}<p^{\prime} v_{x}^{\prime}>=0
$$

déduite des relations (4), il est aisé de vérifier que :

$$
<\mathrm{I}>+<\mathrm{II}>=2 \varepsilon<\bar{v}_{x}>\alpha h l .
$$

b) A partir de rot $\langle\mathbf{E}\rangle=0$ et des relations (4), on obtient :

$$
\begin{aligned}
& <E_{z}>=\text { Cte }=<\bar{E}_{z}> \\
& <E_{x}>=\text { Cte }=0 .
\end{aligned}
$$

De façon à calculer III + IV évaluons $\langle\mathbf{E} \wedge \breve{\mathbf{H}}\rangle$

$$
\langle\mathbf{E} \wedge \breve{\mathbf{H}}\rangle=\langle\mathbf{E}\rangle \wedge\left\langle\breve{\mathbf{H}^{\prime}}\right\rangle+\left\langle\mathbf{E}^{\prime} \wedge{\breve{\mathbf{H}^{\prime}}}^{\prime}\right\rangle
$$

soit d'après (38)

$$
(<\mathbf{E}>\wedge<\breve{\mathbf{H}}\rangle)_{y}=\left\langle E_{z}\right\rangle\left\langle H_{x}\right\rangle=\left\langle\bar{E}_{z}\right\rangle\left\langle H_{x}\right\rangle .
$$

Dans l'isolant en $|y|>\varepsilon$

$$
-\operatorname{div}\left\langle\mathbf{E}^{\prime} \wedge{\breve{\mathbf{H}^{\prime}}}^{\prime}\right\rangle=\left\langle\mathbf{E}^{\prime} \cdot \operatorname{rot} \overleftrightarrow{\mathbf{H}^{\prime}}\right\rangle-\left\langle\overleftrightarrow{\mathbf{H}^{\prime}} \cdot \overleftrightarrow{\operatorname{rot}} \mathbf{E}^{\prime}\right\rangle
$$

soit encore, avec

$$
\begin{gathered}
\operatorname{rot} \breve{\mathbf{H}}^{\prime}=\varepsilon_{0} \frac{\partial \mathbf{E}^{\prime}}{\partial t} \quad \text { et } \quad \operatorname{rot} \mathbf{E}^{\prime}=-\frac{\partial \breve{\mathbf{B}}^{\prime}}{\partial t}, \\
-\operatorname{div}\left\langle\mathbf{E}^{\prime} \wedge \breve{\mathbf{H}}^{\prime}\right\rangle=-\frac{\partial}{\partial y}\left(\left\langle E^{\prime} \wedge H^{\prime}\right\rangle\right)_{y}=\frac{\mu_{0}}{2}\left\langle\frac{\partial H^{\prime 2}}{\partial t}\right\rangle+\frac{\varepsilon_{0}}{2}\left\langle\frac{\partial E^{\prime 2}}{\partial t}\right\rangle=0
\end{gathered}
$$


les fluctuations devant s'annuler pour $|y| \rightarrow \infty$, on en déduit pour $|y|>\varepsilon$ :

$$
\left.<\left(\mathbf{E}^{\prime} \wedge \breve{\mathbf{H}}^{\prime}\right)_{y}\right\rangle=\left\langle H_{x}^{\prime} E_{z}^{\prime}-E_{x}^{\prime} H_{z}^{\prime}\right\rangle=0 .
$$

Les fluctuations étudiées font intervenir les composantes de $\mathbf{E}$ et $\breve{\mathbf{H}}$ continues à l'interface isolant, métal liquide, et par conséquent dans le métal, $\left\langle\mathbf{E}^{\prime} \wedge \breve{\mathbf{H}}^{\prime}\right\rangle_{y}=0$ au niveau des parois, en $y= \pm \varepsilon \mp 0$.

D'autre part, d'après $\langle\mathbf{j}\rangle=\operatorname{rot}\langle\overrightarrow{\mathbf{H}}\rangle$,

$$
\mathfrak{J}=\int_{-\varepsilon}^{+\varepsilon}<j_{z}>\mathrm{d} y=\left(<H_{x}>\right)_{y=-\varepsilon}-\left(<H_{x}>\right)_{y=+\varepsilon} .
$$

Les éq. (40), (41), (43) et (44) permettent donc d'écrire :

$$
\left[<(\mathbf{E} \wedge \breve{\mathbf{H}})_{y}>\right]_{y=+\varepsilon}-\left[<(\mathbf{E} \wedge \breve{\mathbf{H}})_{y}>\right]_{y=-\varepsilon}=-\left\langle\bar{E}_{z}>J\right.
$$

avec

$$
\left\langle E_{z}>=\frac{\left\langle j_{z}>\right.}{\gamma}-<(\mathbf{V} \wedge \breve{\mathbf{B}})_{z}>=\frac{J}{2 \varepsilon \gamma}-B_{0}<\bar{v}_{x}>-\left(<\overline{B_{y}^{\prime} v_{x}^{\prime}}>-<\overline{B_{x}^{\prime}} \overline{v_{y}^{\prime}}>\right)\right.
$$

soit

$$
<\mathrm{III}>+<\mathrm{IV}>=h l\left[-\frac{\mathfrak{J}^{2}}{2 \varepsilon \gamma}+J B_{0}<\bar{v}_{x}>+\mathfrak{J}\left(\left\langle\overline{B_{y}^{\prime} v_{x}^{\prime}}>-<\overline{B_{x}^{\prime} v_{y}^{\prime}}>\right)\right]\right.
$$
Il vient :

c) Le calcul de $\left(\langle\mathbf{V}\rangle \cdot\langle\tilde{\boldsymbol{\sigma}}\rangle_{y}\right)$ pour $y= \pm \varepsilon$ s'effectue très simplement à l'aide des relations (5) et (3).

$$
\begin{aligned}
{\left[(<\mathbf{V}><\tilde{\boldsymbol{\sigma}}>)_{y}\right]_{y= \pm \varepsilon}=\left[\sum_{k}<v_{k}><\sigma_{i k}>\right]_{y= \pm \varepsilon} } & =\left[\left\langle v_{x}><\sigma_{y x}>\right]_{y= \pm \varepsilon}\right. \\
& =\left[\eta<v_{x}>\frac{\left.\partial<v_{x}\right\rangle}{\partial y}\right]_{y= \pm \varepsilon}
\end{aligned}
$$

Soit, grâce à (20)

$$
<\mathrm{V}>+<\mathrm{VI}>=\left(<\tau_{\mathrm{p}_{1}}>U_{1}-<\tau_{\mathrm{p}_{2}}>U_{2}\right) h l .
$$

Des éq. (33), (35), (37), (46) et (48) on tire l'expression générale des dissipations totales par unité de surface du plan $x O z$ :

$$
\begin{aligned}
\frac{\leq P_{\mathrm{d}}}{S}=\frac{<P_{\mathrm{d}}>}{h l}=\frac{\mathfrak{J}^{2}}{2 \varepsilon \gamma}- & <\bar{v}_{x}>B_{0} \mathfrak{J}-<\tau_{\mathrm{p}_{1}}>U_{1}+<\tau_{\mathrm{p}_{2}}>U_{2} \\
& -2 \alpha<\bar{v}_{x}>\varepsilon-\mathfrak{J}\left[<\overline{B_{y}^{\prime} v_{x}^{\prime}}>-<\overline{B_{x}^{\prime} v_{y}^{\prime}}>\right]
\end{aligned}
$$

ou encore grâce à (19), (24) et (44)

$$
\begin{gathered}
\frac{\left\langle P_{\mathrm{d}}>\right.}{S}=\frac{\mathfrak{J}^{2}}{2 \varepsilon \gamma}-<\tau_{\mathrm{p}_{1}}>.\left(U_{1}-<\bar{v}_{x}>\right)-<\tau_{\mathrm{p}_{2}}>\left(<\bar{v}_{x}>-U_{2}\right)+J\left(<B_{x}^{\prime} v_{y}^{\prime}>-<B_{y}^{\prime} v_{x}^{\prime}>\right) \\
-\mu_{0}\left[\left(<H_{x}^{\prime} H_{y}^{\prime}>\right)_{y=+\varepsilon}-\left(<H_{x}^{\prime} H_{y}^{\prime}>\right)_{y=-\varepsilon}\right]<\bar{v}_{x}>
\end{gathered}
$$

avec

$$
\left\langle\tau_{\mathrm{p}_{1}}>-\left\langle\tau_{\mathrm{p}_{2}}\right\rangle=-2 \alpha \varepsilon-B_{0} J+\mu_{0}\left[\left(<H_{x}^{\prime} H_{y}^{\prime}\right\rangle\right)_{y=+\varepsilon}-\left(\left\langle H_{x}^{\prime} H_{y}^{\prime}\right\rangle\right)_{y=-\varepsilon}\right] .
$$

Remarque : l'expression (50) ne permet pas de séparer les pertes par effet Joule des pertes par viscosité. Seules apparaissent les pertes par effet Joule dues au courant extérieur traversant le dispositif.

1.2. DesCription Qualitative De La turbulenCe. - Nous considérerons dans ce paragraphe des écoulements à parois fixes $\left(U_{1}=U_{2}=0\right)$, en régime turbulent, hydrodynamiques $\left(B_{0}=0\right)$ et magnétohydrodynamiques $\left(B_{0} \neq 0\right)$ [cf. Fig. 1].

Les équations établies au paragraphe précédent sont rigoureuses en ce sens qu'elles permettent de connaître les dissipations de puissance dans un fluide lorsqu'on connaît les fluctuations des différentes grandeurs et les contraintes pariétales. Pour obtenir des renseignements sur les fluctuations et les contraintes pariétales, deux approches sont possibles : 
- un procédé expérimental de mesure directe; ainsi Murgatroyd [7], Hartmann et Lazarus [8] ont obtenu les contraintes pariétales d'un écoulement M. H. D. turbulent entre parois fixes ;

- un procédé théorique d'analyse qualitative, utilisant essentiellement des considérations dimensionnelles comme celles décrites par Landau et Lifschitz [9] pour des écoulements hydrodynamiques turbulents.

1.2.1 Rappel des considérations de Landau et Lifschitz sur les écoulements hydrodynamiques $\left(B_{0}=0\right)$ turbulents. - Nous désignons par

$$
\mathbf{V}^{\prime}=\langle\mathbf{V}\rangle-\mathbf{V}
$$

la partie fluctuante de la vitesse du fluide. Landau et Lifschitz dans leur ouvrage Fluids Mechanics page (116) indiquent que dans le cas d'écoulements hydrodynamiques turbulents :

1) On peut rendre compte de $\mathbf{V}^{\prime}$ par la superposition de mouvements turbulents de différentes échelles. L'échelle $\lambda_{\alpha}$ d'un mouvement turbulent $\alpha$ est l'ordre de grandeur des distances sur lesquelles une composante quelconque ( $v_{\alpha i}^{\prime}$ par exemple), de la vitesse $\mathbf{V}_{\alpha}^{\prime} \mathrm{du}$ mouvement varie notablement. L'échelle la plus grande, repérée par l'indice $G$, est dite échelle fondamentale $\left(\lambda_{\mathrm{G}}=l\right)$. Pour des écoulements entre deux plans parallèles, cette échelle $l$ est de l'ordre de grandeur de la distance $2 \varepsilon$ entre les parois $(l \approx 2 \varepsilon)$.

2) Les sources extérieures cèdent continuellement de l'énergie aux mouvements turbulents de grande échelle qui en cèdent eux-mêmes aux mouvements de plus petite échelle. Cette énergie n'est dissipée par viscosité sous forme thermique que pour les mouvements turbulents d'échelle suffisamment petite. L'ordre de grandeur de la dissipation moyenne peut donc être déterminé à partir des caractéristiques des mouvements de grande échelle.

3) A un instant donné, la variation d'une grandeur quelconque $f$ dans une région dont les diamètres sont de l'ordre de $L$ est désignée par $\Delta f(L)$; cette variation $\Delta f(L)$ est simplement la différence entre la valeur maximale et la valeur minimale de $f$ observée dans cette région. La variation $\Delta v_{i}^{\prime}(L)$ de la vitesse fluctuante est d'autant plus importante que $L$ est plus grand; la valeur maximale $\Delta v_{i}^{\prime}(l)$ est comparable à la variation de la vitesse moyenne $\Delta<v_{x}>(l)$ à la même échelle $l$ :

$$
\Delta v_{i}^{\prime}(l) \approx \Delta<v_{x}>(2 \varepsilon) \text {. }
$$

4) Les grandeurs caractéristiques des mouvements de grande échelle étant $\rho$ (masse volumique), $2 \varepsilon$ et $\Delta<v_{x}>(2 \varepsilon)$, la seule combinaison ayant les dimensions d'une densité volumique de puissance, soit :

$$
\pi=\rho \frac{\left[\Delta<v_{x}>(2 \varepsilon)\right]^{3}}{2 \varepsilon}
$$

fournit l'ordre de grandeur de la densité volumique de la puissance dissipée dans un écoulement hydrodynamique turbulent. Dans ces types d'écoulement, les relevés expérimentaux des distributions de $\left\langle v_{x}\right\rangle(y)$ dans la direction $O y$ perpendiculaire aux parois, montrent que les profils de vitesse sont très aplatis $\left(\left\langle v_{x}\right\rangle\right.$ est quasi constante dans la totalité de la largeur $2 \varepsilon$ ). Pour un écoulement hydrodynamique entre parois immobiles, on a donc :

$$
\Delta<v_{x}>(2 \varepsilon) \approx<\bar{v}_{x}>
$$

et l'expression de la densité volumique de puissance dissipée $[\mathrm{cf} .(52)]$ devient :

$$
\pi=\frac{\rho<\bar{v}_{x}>^{3}}{2 \varepsilon}
$$

1.2.2 Ecoulements magnétohydrodynamiques $\left(B_{0} \neq 0\right)$ turbulents à parois fixes. - Dans le cas d'écoulements magnétohydrodynamiques turbulents, les mesures de dissipations dans des conduites à parois fixes montrent que les pertes sont, en régime turbulent, peu différentes, à vitesse moyenne donnée $\left\langle\bar{v}_{x}\right\rangle$, des pertes en régime hydrodynamique. Guidés par ces remarques, nous avons admis que l'analyse précédente de la turbulence en régime hydrodynamique est encore valable en régime magnétohydrodynamique : de l'énergie est transférée constamment, des mouvements de grande échelle vers les mouvements de petites échelles.

Nous utiliserons au paragraphe 1.4.2 les résultats de l'analyse qualitative précédente pour décrire les écoulements M. H. D. à parois mobiles, et les relier quantitativement aux résultats de la théorie semiempirique développée par Harris [10].

1.3 CONDITIONS DE PASSAGE DU RÉGIME LAMINAIRE AU RÉGIME TURBULENT POUR DES ÉCOULEMENTS M. H. D. $(B \neq 0)$. - Nous utiliserons les notations suivantes :

$$
R=\rho \frac{\bar{v}_{x} 4 \varepsilon}{\eta} \text { ou } \rho \frac{<\bar{v}_{x}>4 \varepsilon}{\eta}
$$

nombres de Reynolds de l'écoulement étudié lorsque le régime est respectivement laminaire ou turbulent.

$$
M=B_{0} \sqrt{\frac{\gamma}{\eta}} \varepsilon
$$

nombre de Hartmann correspondant.

Nous définirons d'autre part de façon classique un coefficient de friction $f_{j}(j=1,2)$ relatif à chacune des parois mobiles (1) et (2) par la relation [cf. (18)] :

$$
f_{j}=\frac{8\left|T_{\mathrm{p} j}\right|}{\rho\left(U_{j}-\bar{v}_{x}\right)^{2}} \quad \text { ou } \quad \frac{8 \mid<T_{\mathrm{p} j}>1}{\rho\left(U_{j}-<\bar{v}_{x}>\right)^{2}}
$$

en régime laminaire ou turbulent, respectivement.

Pour un écoulement entre deux parois immobiles, le calcul de $f$ relatif au régime laminaire conduit à :

$$
f_{1}=f_{2}=f=\frac{32 M^{2} \text { th } M}{R(M-\text { th } M)}
$$

soit $f=96 / R$ pour $B_{0}=0$. 
Expérimentalement la transition entre le régime laminaire et le régime turbulent a lieu pour une valeur particulière $f_{\mathrm{c}}$ du coefficient de friction. Cette valeur $f_{\mathrm{c}}$ donnée par différents expérimentateurs [8], [10] est

$$
f_{1 \mathrm{c}}=f_{2 \mathrm{c}}=f_{\mathrm{c}} \simeq 3 \times 10^{-2} .
$$

Les éq. (54) (57) et (58) permettent donc de déterminer la valeur critique de transition $v_{\mathrm{c}}$ de la vitesse $\bar{v}_{\boldsymbol{x}}$ :

$$
v_{\mathrm{c}}=\frac{8 \eta}{\rho \cdot f_{\mathrm{c}} \cdot \varepsilon} \frac{M^{2} \text { th } M}{M-\operatorname{th} M} \simeq \frac{800}{3} \frac{\eta}{\rho \varepsilon} \frac{M^{2} \text { th } M}{M-\operatorname{th} M} .
$$

La courbe de la figure 2 représente les différents domaines de variation de $\left|\left\langle\bar{v}_{x}\right\rangle\right|$ ou $\left|\bar{v}_{x}\right|$ et de $B_{0}$, correspondant à un régime turbulent ou laminaire pour $\eta, \rho, \gamma$ et $\varepsilon$ donnés. Pour $M>2$, l'expression (59) se simplifie et donne avec une très bonne approximation :

$$
v_{\mathrm{c}} \# \frac{800}{3} \frac{\eta}{\rho \varepsilon} \frac{M^{2}}{M-1} .
$$

Pour la plupart des cas pratiques pour lesquels on a $M>10$ ou 20 , on peut considérer plus simplement

$$
v_{\mathrm{c}} \# \frac{800}{3} \frac{\eta}{\rho \varepsilon} M \# \frac{800(\gamma \eta)^{1 / 2}}{3 \rho} B_{0} .
$$

$\begin{array}{ccc}\text { Métaux } & \stackrel{\rho}{\rho} & \Omega^{-1^{\gamma} \mathrm{m}^{-1}} \\ \text { liquides } & \mathrm{kg} \cdot \mathrm{m}^{-3} & - \\ \overline{\mathrm{Hg}} & 1,36 \times 10^{4} & 10^{6} \\ \mathrm{Hg} \mathrm{In} & \sim 10^{4} & 2,5 \times 10^{6} \\ (\mathrm{Na} \mathrm{K}) & 0,87 \times 10^{3} & 2,3 \times 10^{6} \\ 78 \% & & \end{array}$

Pour des écoulements entre parois immobiles, le changement de région se produit quand la valeur absolue $\left|\bar{v}_{x}\right|$ de la vitesse moyenne atteint la valeur critique $v_{\mathrm{c}}$. Il en résulte que lorsque les deux parois se déplacent à la même vitesse $U$, la transition apparaît pour

$$
\left|\bar{v}_{x}-U\right|=v_{\mathrm{c}} .
$$

En écoulement magnétohydrodynamique, même en régime laminaire, la vitesse $v_{x}$ est quasi constante sur l'épaisseur du canal (cette valeur quasi constante étant très peu différente de $\bar{v}_{x}$ ). Les phénomènes de turbulence sont donc liés à la différence entre la vitesse d'une paroi et la vitesse du fluide à son voisinage. Quand les deux parois se déplacent à des vitesses différentes $\left(U_{1} \neq U_{2}\right)$, il est donc normal d'admettre que la turbulence apparaît quand :

$$
\operatorname{Max}\left|\bar{v}_{x}-U_{j}\right|=v_{\mathrm{c}},
$$

la turbulence produite au voisinage de la paroi $j$ [correspondant à la plus grande valeur de $\left|\bar{v}_{x}-U_{j}\right|$ ] s'étendant à tout le fluide.

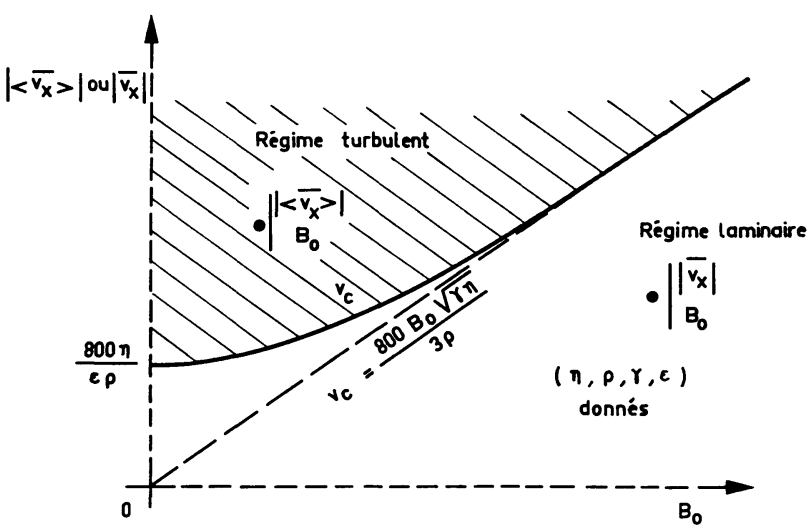

FIG. 2. - Différents domaines correspondant aux régimes laminaires et turbulents en fonction de l'induction extérieure appliquée $B_{0}$, dans le cas de parois fixes.

Sur le tableau ci-après, nous avons reporté pour quelques métaux liquides, les valeurs correspondantes des vitesses critiques $v_{c}$ pour $M>20$ soit :

$$
B_{0}>\frac{20}{\varepsilon} \sqrt{\frac{\eta}{\gamma}} \text {. }
$$

$$
\begin{gathered}
\stackrel{\eta}{(60} \stackrel{\circ}{\circ}) \mathrm{Pl} \\
(\mathrm{MKSA}) \\
- \\
1,5 \times 10^{-3} \\
1,5 \times 10^{-3} \\
0,55 \times 10^{-3}
\end{gathered}
$$$$
\begin{gathered}
\frac{v_{\mathrm{c}}}{B_{0}}=\frac{800(\gamma \eta)^{1 / 2}}{3 \rho} \mathrm{m}^{3} \cdot \mathrm{s}^{-1} \cdot \mathrm{W}_{\mathrm{b}}^{-1} \\
\overline{0,75} \\
1,6 \\
19,6
\end{gathered}
$$

1.4 Evaluation Des CONTRaintes pariétales $<T_{p_{1}}>$ ET $<T_{p_{2}}>$ POUR LE CALCUL DES DISSIPATIONS. - Pour simplifier la suite de l'exposé, nous supposons que, dans l'expression (18) de la contrainte pariétale, le terme $\mu_{0}\left\langle H_{x}^{\prime} H_{z}^{\prime}\right\rangle_{y \pm \varepsilon}$ est négligeable. Remarquons que dans le cas où l'écoulement a lieu entre des parois conductrices recouvertes d'une couche d'isolant, on peut montrer que la valeur de $\mu_{0}\left\langle H_{x}^{\prime} H_{z}^{\prime}\right\rangle$ est d'autant plus faible que la couche isolante est plus mince. Par ailleurs les différentes expériences [7], [8], [10], [12], [13], [14], [15], [16] sont toujours dépouillées et interprétées sans faire intervenir la présence ou l'absence d'un conducteur sous la couche isolante. Ceci donne un argument expérimental pour, en première approximation, négliger en toute circonstance le terme incriminé.

1.4.1 Ecoulements plans à parois fixes, avec $\mathfrak{J}=0$. - Dans le cas de ces expériences $\left(U_{1}=U_{2}=0\right.$, $\mathfrak{J}=0$ ) la symétrie de l'écoulement par rapport au plan $x O z$ entraîne :

$$
\left(<H_{x}>\right)_{ \pm \varepsilon}=0
$$


ainsi que

$$
\left|\left(\frac{\partial<v_{x}>}{\partial y}\right)_{+\varepsilon}\right|=\left|\left(\frac{\partial<v_{x}>}{\partial y}\right)_{-\varepsilon}\right| .
$$

L'expression (18) de la contrainte pariétale devient ainsi, en négligeant $\left.\mu_{0}<H_{x}^{\prime} H_{z}^{\prime}\right\rangle_{ \pm \varepsilon}$

$$
<T_{\mathrm{p}_{2}^{1}}>=\eta\left(\frac{\partial<v_{x}>}{\partial y}\right)_{y=\mp \varepsilon}=<\tau_{\mathrm{p}_{2}^{1}}>
$$

la fonction $\left\langle v_{x}\right\rangle(y)$ étant paire

$$
\left|<\tau_{\mathbf{p}_{1}}>\right|=\left|<\tau_{\mathbf{p}_{2}}>\right|=<\tau_{H_{a}}>
$$

et donc

$$
\left|<T_{\mathbf{p}_{1}}>\right|=\left|<T_{\mathbf{p}_{2}}>\right|=<\tau_{H_{\mathrm{a}}}>\text {. }
$$

Les études théoriques et expérimentales de Murgatroyd [7], Hartmann et Lazarus [8] et Harris [10] permettent pour des écoulements magnétohydrodynamiques plans à parois fixes $\left(U_{1}=U_{2}=0\right)$ d'obtenir une détermination $\left\langle\tau_{H_{a}}\right\rangle$ de la contrainte pariétale.

L'expression (50) permet d'exprimer les dissipations par unité de surface des parois sous la forme

$$
\begin{aligned}
\frac{\left\langle P_{\mathrm{d}}\right\rangle}{S}=\left(<\tau_{\mathrm{p}_{1}}>\right. & \left.-<\tau_{\mathrm{p}_{2}}>\right) .<\bar{v}_{x}>= \\
& =2<\tau_{H_{\mathrm{a}}}>\left|<\bar{v}_{x}>\right| .
\end{aligned}
$$

En régime laminaire l'expression de la contrainte $\tau_{H_{a}}$ est classique cf. : (61), (56), (57) et (54) :

$$
<\tau_{H_{\mathrm{a}}}>=\tau_{H_{\mathrm{a}}}=\frac{\eta \bar{v}_{x}}{\varepsilon} \frac{M^{2} \text { th } M}{M-\operatorname{th} M}
$$

soit, pour le cas particulier où $B_{0}=0$,

$$
\tau_{H_{\mathrm{a}}}=\frac{3 \eta \bar{v}_{x}}{\varepsilon}
$$

En régime turbulent $\left\langle\tau_{H_{\mathrm{a}}}>\right.$ est donnée par :

$$
<\tau_{H_{\mathrm{a}}}>=\frac{\rho}{8}<\bar{v}_{x}>^{2} \cdot f\left(<\bar{v}_{x}>\right) .
$$

Pour un régime turbulent donné, en absence d'induction magnétique appliquée $B_{0}(\rho, \eta, \varepsilon$ donnés $)$ $f\left(<\bar{v}_{x}>\right)$ est obtenu par la relation :

$$
\frac{1}{\sqrt{f}}-2 \log _{10}(R \sqrt{f})+0,8=0
$$

dite loi de Nikuradse [10]. Dans le cas d'un écoulement M. H. D. turbulent, cette loi doit être remplacée par

$$
\frac{1}{\sqrt{f}}-2 \log _{10}(R \sqrt{f})+0,8=A\left(\frac{R \sqrt{f}}{M^{2}}\right)
$$

où $A\left(\frac{R \sqrt{f}}{M^{2}}\right)$ est une fonction semi-empirique de $\frac{R \sqrt{f}}{M^{2}}$. Toujours dans le cas où les parois délimitant l'écoulement sont fixes, les résultats de l'étude de Harris [11] permettent de déterminer $A\left(\frac{R \sqrt{f}}{M^{2}}\right)$ dont on déduit la courbe $\left\langle\tau_{H_{a}}\right\rangle\left(\left\langle\bar{v}_{x}\right\rangle\right)$ pour un écoulement donné. Nous avons représenté sur la figure 3, l'allure de la variation de la contrainte pariétale $\tau_{H_{\mathrm{a}}}$, où $\left\langle\tau_{\boldsymbol{H}_{\mathrm{a}}}\right\rangle$ en régime laminaire et turbulent compte tenu du critère de transition (59) et des expressions (63) à (66). Ces courbes ont été tracées pour $\rho, \eta, \varepsilon$ donnés avec $B_{0}$ pour paramètre.

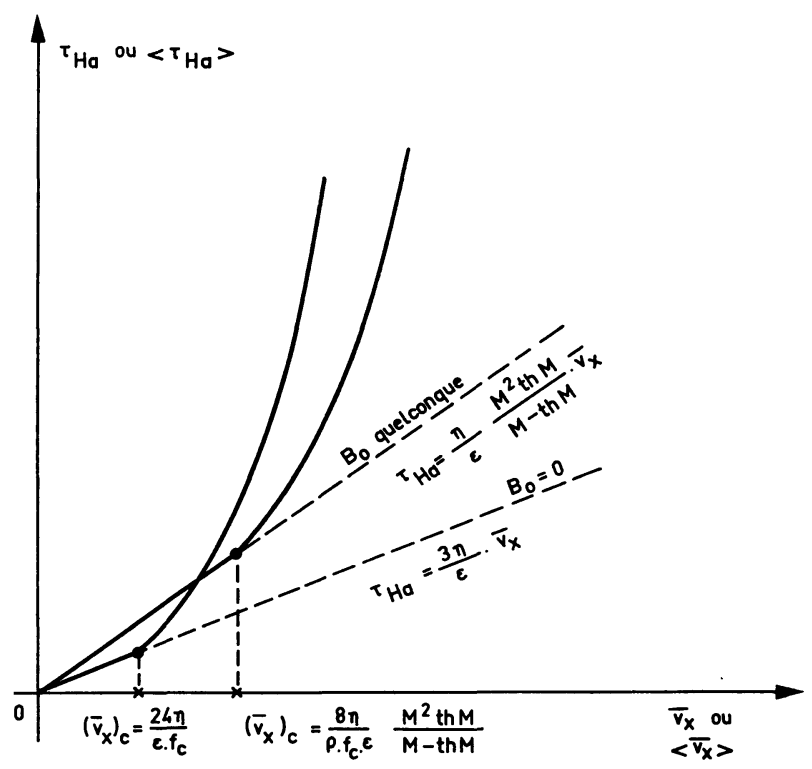

FIG. 3. - Allure de la variation de la contrainte pariétale en fonction de la vitessé de débit de l'écoulement en hydrodynamique $\left(B_{0}=0\right)$ et en magnétohydrodynamique $\left(B_{0} \neq 0\right)$ dans le cas de parois fixes.

1.4.2 Evaluation des contraintes pariétales dans les écoulements à parois mobiles dans le cas général où $B_{0}$, $U_{1}$ et $U_{2}$ sont quelconques : Les expressions (49), (50) et (51) sont fortement complexes, aussi allons-nous chercher à les simplifier. La remarque que nous avons déjà effectuée au paragraphe 1.4 sur le terme $\left.\mu_{0}\left(<H_{x}^{\prime} H_{y}^{\prime}\right\rangle\right)_{ \pm \varepsilon}$ nous permet de négliger ce produit de fluctuations. Comparons maintenant, dans le cas simple d'un écoulement entre parois fixes où la mise en vitesse du fluide s'effectue par l'intermédiaire d'un gradient de pression, les deux termes :

$$
A_{1}=\left\langle\overline{B_{y}^{\prime} v_{x}^{\prime}}\right\rangle-\left\langle\overline{B_{x}^{\prime} v_{y}^{\prime}}\right\rangle
$$

et

$$
A_{2}=B_{0}<\bar{v}_{x}>
$$

Il vient

$$
A_{1}<2 \sqrt{\left\langle\overline{\left|B^{\prime}\right|^{2}}\right.}>\cdot \sqrt{<\overline{\left|v^{\prime}\right|^{2}}}>\text {. }
$$

En posant :

$$
u^{*}=\sqrt{\frac{2 \alpha \varepsilon}{\rho}} ; \quad \breve{b}=\frac{\breve{B}}{B_{0}^{\prime}} .
$$


Cela revient à comparer :

$$
2 \sqrt{<\overline{b^{\prime 2}}>} \sqrt{\frac{\left\langle\overline{v^{\prime 2}}\right\rangle}{u^{* 2}}} \text { et } \frac{<\bar{v}_{x}>}{u^{*}} \text {. }
$$

Les résultats expérimentaux tirés de [7], [8], [12], [13] et [14] conduisent à d'une part

$$
20<\frac{\left\langle\bar{v}_{x}>\right.}{u^{*}}<45 \text { soit } \frac{\left\langle\bar{v}_{x}>\right.}{u^{*}} \simeq 30
$$

et d'autre part

$$
\sqrt{\frac{\left\langle\overline{v^{\prime 2}}>\right.}{u^{* 2}}} \lesssim 3
$$

La condition

$$
A_{1} \ll A_{2}
$$

impose donc l'inégalité

$$
\frac{\left.\sqrt{<\overline{\left.B^{\prime}\right|^{2}}}\right\rangle}{B_{0}} \ll 5 .
$$

Nous supposerons dans la suite de l'étude que cette condition est satisfaite. Ceci semble justifié en remarquant :

- d'une part que si des fluctuations d'une telle amplitude existaient, l'observation de la plupart des grandeurs l'aurait montré ;

- et que d'autre part l'inégalité (75) a été obtenue en supposant les différentes grandeurs parfaitement corrélées.

Nous obtenons dans ces conditions et toujours dans le cadre de l'approximation du paragraphe 1.4

$$
\begin{aligned}
\frac{\left\langle P_{\mathrm{d}}\right.}{S} \# \frac{J^{2}}{2 \varepsilon \gamma}- & <\tau_{\mathbf{p}_{1}}>.\left(U_{1}-<\bar{v}_{x}>\right)- \\
& -<\tau_{\mathbf{p}_{2}}>\cdot\left(<\bar{v}_{x}>-U_{2}\right) \\
<\tau_{\mathbf{p}_{1}}>-<\tau_{\mathbf{p}_{2}}> & =-2 \frac{\partial<p>}{\partial x} \varepsilon-B_{0} J .
\end{aligned}
$$

Pour un écoulement donné $-\left(B_{0}, \mathfrak{J}, U_{1}, U_{2}, \varepsilon, \gamma, \rho, \eta\right.$, $\partial\langle p\rangle / \partial x$ connus) - les éq. (76) et (77) permettraient, connaissant la variation des contraintes pariétales en fonction de $\left\langle\bar{v}_{x}\right\rangle$, de calculer la vitesse moyenne $\left\langle\bar{v}_{x}\right\rangle$ et les dissipations moyennes par unité de surface. Ces contraintes pariétales pourraient être déterminées à l'aide d'une méthode semi-empirique voisine de celle utilisée par Harris [10] dans le cas de parois fixes, au prix d'un travail expérimental rendu beaucoup plus délicat par la nécessité de disposer d'une paroi mobile.

Dans un travail antérieur [17] nous avions déterminé les puissances dissipées dans un écoulement de ce type, en supposant que la contrainte $\tau_{\mathrm{p} j}$ sur la paroi $j$ était égale à celle qui aurait été observée dans un écoulement à la vitesse moyenne $\left|U_{j}-<\bar{v}_{x}\right\rangle \mid$ s'effectuant entre parois fixes, soit

$\left|<\tau_{\mathrm{p} j}>\right|=<\tau_{H_{\mathrm{a}}}>\left(\left|U_{j}-<\bar{v}_{x}>\right|\right) \quad(j=1,2)$

Les dissipations calculées à partir des expressions (76) et (78) sont environ deux fois plus faibles que les dissipations mesurées dans [17].

Pour rendre compte plus précisément des résultats expérimentaux, nous avons formulé une hypothèse fondée sur l'analyse dimensionnelle du paragraphe 1.2 [éq. (53)]. D'après ces considérations et en remarquant qu'en régime turbulent $\left\langle v_{x}\right\rangle(y)$ est quasi constant sur l'épaisseur de la veine $(2 \varepsilon)$ et voisin de $\left\langle\bar{v}_{x}\right\rangle$, nous faisons l'hypothèse que les pertes ne peuvent dépendre que de $\left|U_{1}-<\bar{v}_{x}>\right|$ et de $\left|U_{2}-<\bar{v}_{x}\right\rangle \mid$ pour $B_{0}, \varepsilon, \rho, \eta, \gamma, \partial<\mathrm{p}>/ \partial x, U_{1}$ et $U_{2}$ donnés. Comme nous avons vu d'autre part que la turbulence est liée à la valeur maximale de $\left|U_{j}-\left\langle\bar{v}_{x}\right\rangle\right|$ nous déduisons de notre hypothèse la proposition suivante :

"Pour un écoulement de géométrie donnée pour lequel $B_{0}$ est fixé, et quelle que soit la vitesse relative des parois, les dissipations moyennes dans l'ensemble de la veine fluide constituent - (dans le cas d'un régime turbulent et dans la limite des grands nombres de Reynolds) - une fonction biunivoque du plus grand des modules des vitesses relatives

$$
\left|U_{j}-<\bar{v}_{x}>\right|(j=1,2)
$$

entre le fluide et l'une ou l'autre des parois. »

Il n'est possible de justifier cette proposition que par le bon accord entre les résultats théoriques et expérimentaux auxquels elle conduit, résultats que nous examinerons plus loin. Cependant dans un cas particulier simple, d'un écoulement hydrodynamique de Couette, plan parallèle pour lequel

$$
\left\langle\bar{v}_{x}>-U_{1}=-\left\langle\bar{v}_{x}\right\rangle+U_{2} \text { avec } U_{1}=-U_{2}\right.
$$

il nous a été possible, à l'aide de résultats expérimentaux donnés par [18] de tester la validité de notre hypothèse : la comparaison des coefficients de frictions ainsi calculés et des coefficients mesurés montre un bon accord pour les grands nombres de Reynolds (correspondant aux hypothèses de calcul) tandis que l'erreur relative est de $25 \%$ environ au voisinage de la transition laminaire-turbulent.

Une discussion de cette hypothèse peut cependant être engagée de la façon suivante :

a) $\mathrm{Si}\left|U_{1}-<\bar{v}_{x}\right\rangle \mid$ et $\left|U_{2}-<\bar{v}_{x}\right\rangle \mid$ sont du même ordre de grandeur, on ne fait pas une grosse erreur en les remplaçant par le plus grand des deux.

b) S'ils sont très différents, les vitesses du mouvement pulsatoire de grande échelle sont, en reprenant le raisonnement de Landau et Lifschitz de l'ordre de grandeur des variations de la vitesse moyenne, variations qui correspondent à la valeur maximale de $\left|U_{j}-\left\langle v_{x}\right\rangle\right|$. La dissipation totale étant déterminée 
par les caractéristiques du mouvement pulsatoire de grande échelle, on peut effectivement s'attendre à ce qu'elle ne dépende que de $\operatorname{Max}\left|U_{j}-\left\langle v_{x}\right\rangle\right|$.

Dans le cas général, les dissipations pourront donc être calculées, une fois la vitesse moyenne $\bar{v}_{x}$ déterminée, à l'aide des résultats obtenus dans le cas d'écoulements parallèles entre deux plans fixes pour $B_{0}$ et $\varepsilon$ identiques. Elles auront pour expression

$$
\begin{gathered}
\quad \frac{P>}{S}=\frac{<P_{\mathrm{d}}>}{S}-\frac{J^{2}}{2 \varepsilon \gamma}=2<\tau_{H_{\mathrm{a}}}>\times \\
\times\left(\operatorname{Max}\left|U_{j}-<\bar{v}_{x}>\right|\right) \cdot \operatorname{Max}\left|U_{j}-<\bar{v}_{x}>\right|
\end{gathered}
$$

expression dans laquelle $\operatorname{Max}\left|U_{j}-\left\langle\bar{v}_{x}\right\rangle\right|$ désigne la plus grande valeur absolue prise par $U_{j}-\left\langle\bar{v}_{x}\right\rangle$ avec $j=1,2$.

Les contraintes pariétales étant des fonctions croissantes des vitesses relatives $U_{j}-<\bar{v}_{x}>$ (cf. Fig. 3), l'hypothèse précédente conduit à des dissipations calculées plus élevées que celles obtenues avec l'hypothèse traduite par l'éq. (78). Cet accroissement a pour expression

$$
\begin{aligned}
\frac{\langle\Delta p>}{S} & =2<\tau_{H_{\mathrm{a}}}>\left(\operatorname{Max}\left|U_{j}-<\bar{v}_{x}>\right|\right) \times \\
& \times \operatorname{Max}\left|U_{j}-<\bar{v}_{x}>\right| \\
& -<\tau_{H_{\mathrm{a}}}>\left(\left|U_{1}-<\bar{v}_{x}>\right|\right) \cdot\left(\left|U_{1}-<\bar{v}_{x}>\right|\right) \\
& -<\tau_{H_{\mathrm{a}}}>\left(\left|U_{2}-<\bar{v}_{x}>\right|\right) \cdot\left(\left|U_{2}-<\bar{v}_{x}>\right|\right) .
\end{aligned}
$$

La détermination de la vitesse moyenne $\left\langle\bar{v}_{x}\right\rangle$ pose encore un problème. En effet, pour un écoulement de géométrie donnée où l'on connaît les forces extérieures, le système constitué par l'éq. (77) et par la relation déduite des expressions (76) et (79) à savoir :

$$
\begin{aligned}
-< & \tau_{\mathrm{p}_{1}}>\cdot\left(U_{1}-<\bar{v}_{x}>\right)-<\tau_{\mathrm{p}_{2}}>\times \\
& \times\left(<\bar{v}_{x}>-U_{2}\right)=2<\tau_{H_{\mathrm{a}}}> \\
& \times\left(\operatorname{Max} \mid U_{j}-<\bar{v}_{x}>1\right) . \operatorname{Max}\left|U_{j}-<\bar{v}_{x}>\right|
\end{aligned}
$$

ne permet pas de résoudre complètement le problème, qui comporte les trois inconnues $\left\langle\tau_{\mathbf{p}_{1}}\right\rangle,\left\langle\tau_{\mathbf{p}_{2}}\right\rangle$ et $\left\langle\bar{v}_{x}\right\rangle$. Nous formulerons donc une hypothèse complémentaire, concernant la valeur de la contrainte sur la paroi la moins rapide (par rapport au mouvement moyen du fluide). Si $l$ désigne cette paroi, nous écrirons :

$$
<\tau_{\mathrm{p}}>=<\tau_{H_{\mathrm{a}}}>\left(U_{l}-<\bar{v}_{x}>\right)
$$

où $l$ est l'indice $j=1$ ou 2 qui rend minimal $\left(U_{j}-\bar{v}_{x}\right)$.

Cette hypothèse nous semble justifiée pour les raisons suivantes :

- l'égalité (81) est vérifiée dans les deux cas limites où d'une part les deux parois se déplacent à la même vitesse par rapport au mouvement moyen du fluide et lorsque d'autre part: $U_{j}=\left\langle\bar{v}_{x}\right\rangle$, puisqu'alors $<\tau_{\mathrm{p} j}>=0$,

- l'accroissement de puissance donnée par (80) indique un accroissement de la turbulence par rapport aux hypothèses traduites par (78) ; l'égalité (81) attribue cet accroissement de turbulence à l'action de la paroi la plus rapide, en affectant à cette paroi la totalité de l'accroissement des contraintes globales sur la veine.

L'hypothèse précédente (82) permet, avec l'aide des éq. (77) et (81) de déterminer la vitesse moyenne $\left\langle\bar{v}_{x}\right\rangle$ et les dissipations pour un écoulement turbulent permanent en moyenne, lorsque les paramètres tels que $\mathfrak{J}, \partial<\mathrm{p}>/ \partial x, U_{1}, U_{2}, \varepsilon$ et $B_{0}$ sont fixés.

2. Application de l'étude précédente au calcul des dissipations M.H.D. dans une machine électrique homopolaire. - Nous rappelons brièvement le principe d'un tel moteur, décrit par ailleurs, [19], [20], [21] qui comprend essentiellement (Fig. 4) :

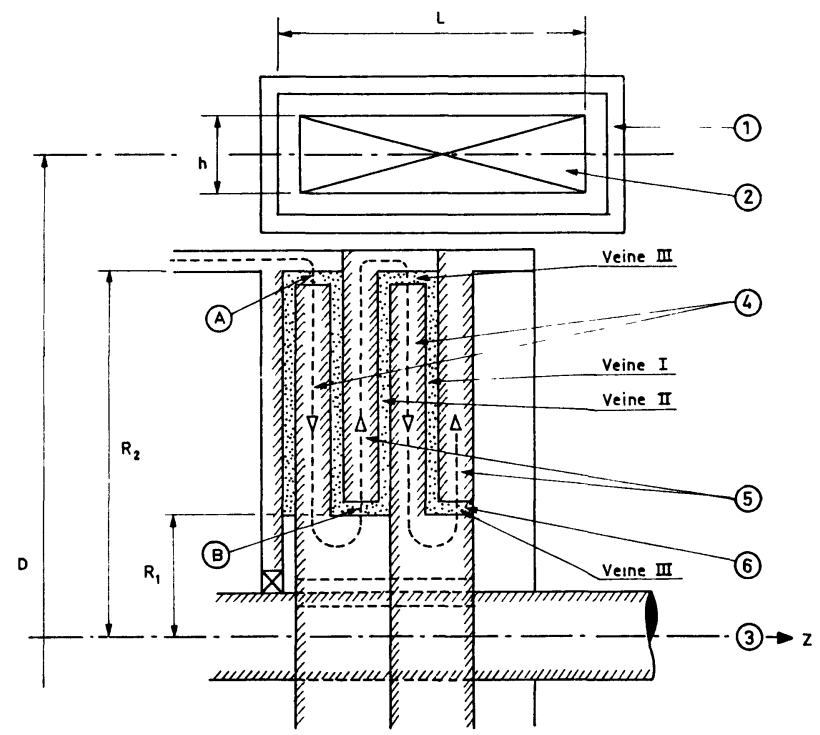

Fig. 4. - Schéma de principe d'une machine homopolaire à disques multiples et inducteur supraconducteur. $777 / 77$ Surfaces isolantes. Surfaces conductrices. Métal liquide conducteur. 1) Appareillage cryogénique. 2) Bobinage inducteur (solénoïde supraconducteur). 3) Axe de rotation. 4) Disques mobiles d'induit. 5) Contre-disques fixes d'induit. 6) Métal liquide conducteur assurant les contacts électriques.

- Un système inducteur composé d'un bobinage supraconducteur ayant pour axe celui de la machine. Ce système crée un champ magnétique à symétrie de révolution autour de l'axe. Les seules composantes du champ inducteur sont axiales et radiales.

- Un induit (à la température ambiante) dont les différents éléments possèdent aussi une symétrie de révolution par rapport à l'axe (arbre de la partie tournante) et qui comprend des conducteurs actifs (disques mobiles) solidaires de l'arbre et isolés électriquement entre eux et des conducteurs de retour (disques fixes) 
solidaires d'un bâti fixe. Les différentes forces électromotrices prenant naissance entre le centre et la périphérie des disques mobiles sont mises en série au moyen de contacts électriques glissants et de disques fixes. Les faces latérales des disques mobiles et fixes sont isolées électriquement.

Le couple mécanique actif de la machine s'exerce sur les disques mobiles, tandis que les conducteurs fixes subissent le couple de réaction. Des contacts électriques glissants de hautes performances sont obtenus en remplissant d'un métal ou alliage liquide, l'espace libre entre les différents disques et contre-disques [22], [23] et [24].

2.1 DESCRIPTION DE LA MÉTHODE DE CONTACT PAR REMPLISSAGE TOTAL. - En se reportant au schéma de la figure 5 , on constate qu'il existe trois catégories de

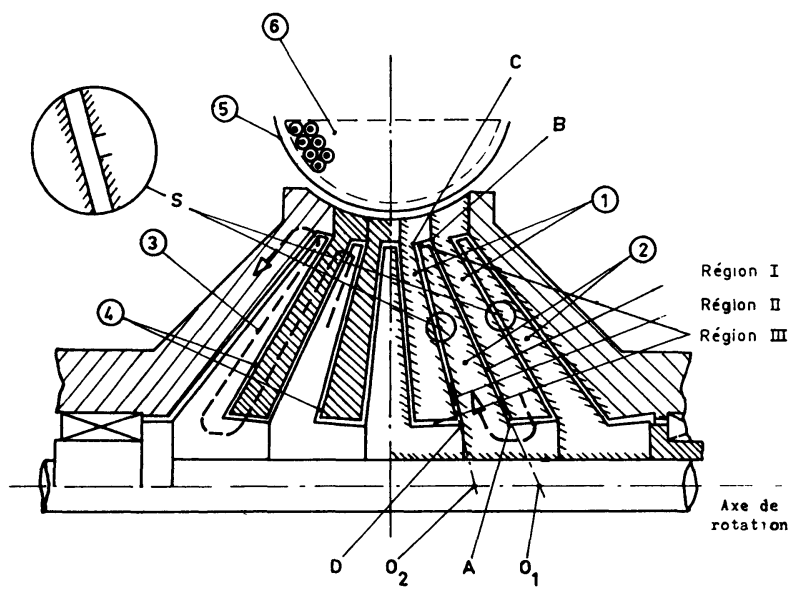

FIG. 5. - Schéma de principe de la machine homopolaire LCIELGEP. La forme tronconique des éléments d'induit résulte d'un calcul d'optimisation en couple volumique de la machine pour une quantité de supraconducteur donnée. $7 \pi 7 \pi 77 \pi$ Surfaces isolées électriquement (les isolants ne sont représentés que sur la moitié droite de la figure). 1) Disques fixes. 2) Disques mobiles. 3) Circulation du courant d'induit I. 4) Métal liquide.

5) Cryostat. 6) Bobinage inducteur supraconducteur.

veines liquides dans chaque cellule élémentaire de l'induit de notre prototype, constituée de deux éléments fixe et mobile. Les conditions électriques aux limites qui leur sont appliquées permettent de distinguer :

- une veine latérale (région I), limitée radialement par les surfaces conductrices des contacts glissants central et périphérique d'un même disque mobile;

- une autre veine latérale (région II) limitée radialement par les contacts glissants central et périphérique d'un même disque fixe ;

- une veine frontale (région III) correspondant au métal liquide situé sous les zones de contacts.

La construction de notre machine est telle que l'on puisse admettre que l'induction magnétique continue $B_{0}$ produite par l'inducteur est perpendiculaire aux parois latérales des veines I et II en tout point de celles- ci. Ces parois sont isolées électriquement, parallèles et distantes de $2 \varepsilon$. Le module de l'induction extérieure appliquée ainsi que la vitesse en un point de la paroi mobile, dépend pour ces écoulements de la distance à l'axe. Les veines frontales traversées par le courant d'alimentation I de la machine diffèrent profondément des veines précédentes.

Nous nous intéresserons dans cet article principalement à l'étude du régime turbulent. Cependant, l'utilisation possible de l'eutectique ( $\mathrm{NaK} 78 \%$ ) conduisant à des vitesses critiques élevées - cf. tableau du paragraphe 1.3 avec par exemple $v_{\mathrm{c}}=80 \mathrm{~m} . \mathrm{s}^{-1}$ pour $B_{0}=5 \mathrm{~T}$ - correspondrait à un régime d'écoulement laminaire.

2.2 Calcul Des dissipations M. H. D. et Des DISTRIBUTIONS DE VITESSES DANS LES VEINES LATÉRALES. - Le schéma de la figure 6 représente la section par un plan axial des différentes veines constituant une cellule élémentaire de la machine.

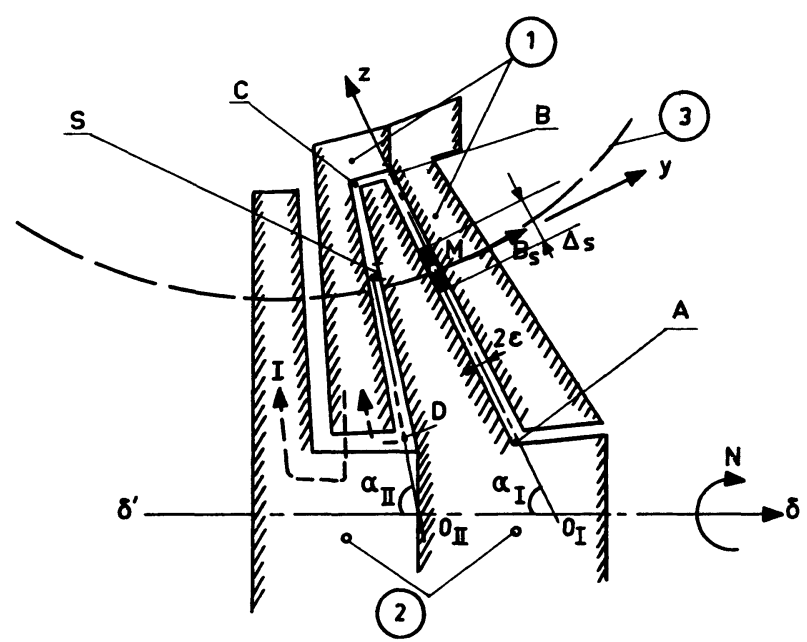

Fig. 6. - Schéma représentant les différentes veines de métal liquide, ainsi que les notations utilisées pour le calcul. 1) Disques fixes. 2) Disques mobiles. 3) Ligne de force du champ magnétique inducteur. $77 / 7 / 7 /$ Surfaces isolées électriquement. Surfaces concuctrices.

La mise en rotation des disques mobiles d'induit fait apparaître dans chacune des régions I et II un courant de circulation global non nul traversant radialement les veines liquides. Soient $i_{\mathrm{I}}$ et $i_{\mathrm{II}}$ ces courants dérivés.

Nous obtiendrons une valeur approchée des dissipations à partir des hypothèses suivantes : (cf. Fig. 6)

a) La veine liquide, d'épaisseur $2 \varepsilon$ constante, est divisée dans le sens radial en anneaux supposés indépendants.

b) Chaque anneau est repéré par l'abscisse $O_{\mathbf{I}} M=s$ du centre $M$ de sa section droite, mesurée suivant une génératrice quelconque du cône d'axe $\delta \delta^{\prime}$; Cette génératrice est équidistante des deux parois limitant latéralement la veine liquide. Soit $M_{\mathbf{y}}$ une direction orthogonale à la précédente perpendiculaire aux parois. La hauteur $\Delta s$ de ces anneaux est supposée 
grande devant $2 \varepsilon$, mais suffisamment faible pour que le module $B_{0}(s)$ de l'induction (perpendiculaire aux parois latérales) et le module $U$ de la vitesse de la paroi soient supposés constants sur $\Delta s$. Tous les anneaux sont traversés en série par le même courant $i_{\mathrm{I}}$ ou $i_{\mathrm{II}}$.

c) On admet en outre que la seule composante importante de la vitesse en tout point du fluide est la composante tangentielle. En effet, supposons qu'en raison des forces centrifuges, il puisse exister en un point de la veine liquide une composante de la vitesse dirigée suivant les génératrices $M_{z}$ du cône limitant chaque veine (cf. Fig. 6) ; sous l'action de l'induction $B_{0}$, cette composante de vitesse produirait une force électromotrice et des courants de direction tangentielle ; la force de Laplace qui en découlerait, s'opposerait aux forces centrifuges de mise en vitesse. Un simple calcul d'ordre de grandeur montre que la composante suivant les génératrices du cône, est négligeable devant la composante tangentielle.

Soit $\bar{v}_{x}(s)$ la composante suivant $M_{x}$ de la vitesse moyenne (sur $y$ et $t$ ), du fluide au point $M\left(O_{\mathrm{I}} M=s\right)$. Le calcul de la distribution de vitesse $\left\langle\bar{v}_{x}\right\rangle$ en fonction de $s$, le long de chacune des veines liquides s'effectue à l'aide des résultats théoriques de l'étude précédente, de la façon suivante :

1) Résolution pour chacun des anneaux liquides composant la veine considérée du système d'équations :

$$
<\tau_{\mathrm{p}_{2}}>-<\tau_{\mathrm{p}_{1}}>-\mathfrak{J}_{\mathrm{I}, \mathrm{II}} \cdot B_{0}=0
$$

(équation dans laquelle le terme $\partial\langle p\rangle / \partial x$ a disparu étant donnée la symétrie de révolution autour de l'axe $\delta^{\prime} \delta$.)

avec

$$
\begin{aligned}
2 & <\tau_{H_{\mathrm{a}}}>\left(\operatorname{Max}\left|U_{j}-<\bar{v}_{x}>\right|\right) \times \\
& \times \operatorname{Max}\left|U_{j}-<\bar{v}_{x}>\right|=-<\tau_{\mathrm{p}_{1}}> \\
& \times\left(U_{1}-<\bar{v}_{x}>\right)-<\tau_{\mathrm{p}_{2}}>\cdot\left(U_{2}-<\bar{v}_{x}>\right)
\end{aligned}
$$

et

$$
\begin{aligned}
& <\tau_{\mathrm{p} j}>\left(\min \left|U_{j}-<\bar{v}_{x}>\right|\right)= \\
& =<\tau_{H_{\mathrm{a}}}>\left(\min \left|U_{j}-<\bar{v}_{x}>\right|\right) .
\end{aligned}
$$

2) Calcul de la distribution de vitesse $\left\langle\bar{v}_{x}\right\rangle$ (s), obtenu par itérations, les vitesses $\left\langle\bar{v}_{x}\right\rangle$ des différents anneaux liquides devant satisfaire les conditions électriques aux limites.

Enfin le calcul des dissipations magnétohydrodynamiques totales pour une veine liquide déterminée, a pour expression :

$$
P_{\mathrm{d}_{\mathrm{I}, \mathrm{II}}}=\sum_{k=1}^{Q}\left(P_{\mathrm{d}_{\mathrm{I}, \mathrm{II}}} / s\right)_{k} \cdot 2 \pi \cdot s_{k} \cdot \sin \alpha_{\mathrm{I}, \mathrm{II}} \cdot(\Delta s)_{k}
$$

où $Q$ est le nombre d'anneaux liquides qui la composent, $s_{k}$ la distance à l'axe $\delta \delta^{\prime}$, mesurée le long de la veine liquide, du $k$-ième anneau, et $(\Delta s)_{k}$ sa largeur (cf. Fig. 6) :

$$
\begin{aligned}
\left(P_{\mathrm{d}_{\mathrm{I}, \mathrm{II}}} / s\right)_{k} & =\left(\frac{\mathfrak{J}_{\mathrm{I}, \mathrm{II}}^{2}}{2 \varepsilon \gamma}\right)_{k}+2<\tau_{H_{\mathrm{a}}}>\times \\
\times & \left(\operatorname{Max}\left|U_{j}-<\bar{v}_{x}>\right|_{k}\right) \cdot \operatorname{Max}\left(U_{j}-<\bar{v}_{x}>\right)_{k} .
\end{aligned}
$$

Nous donnons ci-dessous un exemple d'application de ces équations aux veines de type I, lors d'un fonctionnement de la machine électrique en moteur.

Sachant que pour ce type de veine liquide

$$
\left(U_{1}\right)_{k}=2 \pi \cdot s_{k} \cdot \sin \alpha_{1} \cdot \Omega_{0}
$$

$\left(\Omega_{0}:\right.$ pulsation angulaire de rotation en ${\mathrm{rd} . \mathrm{s}^{-1}}^{-1})$

$$
\left(U_{2}\right)_{k}=0 \text {. }
$$

L'étude précédente conduit aux relations suivantes : - pour

$$
\begin{aligned}
& \left(\frac{U_{1}}{2}\right)_{k}<<\bar{v}_{x}>_{k}<\left(U_{1}\right)_{k} \\
& \frac{i_{\mathrm{I}}}{2 \pi\left(s_{1}\right)_{k} \sin \alpha_{\mathrm{I}}}=2<\tau_{H_{\mathrm{a}}}>\left(<\bar{v}_{x}>_{k}\right)-<\tau_{H_{\mathrm{a}}}>\times \\
& \times\left(U_{1}-<\bar{v}_{x}>\right)_{k} \cdot\left(\frac{U_{1}}{<\bar{v}_{x}>}\right)_{k} ; \\
& <\bar{v}_{x}>_{k}>\left(U_{1}\right)_{k} \\
& \times\left(U_{1}-<\bar{v}_{x}>\right)_{k} \cdot\left(\frac{U_{1}}{\left\langle\bar{v}_{x}>\right.}\right)_{k} .
\end{aligned}
$$

Les conditions électriques pouvant s'écrire

$$
\begin{aligned}
\left|\psi_{\left(s_{1}\right)}-\psi_{\left(s_{2}\right)}\right|= & \left(4 \pi \varepsilon \gamma \sin \alpha_{\mathrm{I}}\right)^{-1} \operatorname{Ln} \frac{s_{2}}{s_{1}} \cdot\left|i_{\mathrm{I}}\right|+ \\
& +\int_{s_{1}}^{s_{2}}|B(s)| \cdot\left|<\bar{v}_{x}>\right|(s) . \mathrm{d} s
\end{aligned}
$$

$\psi_{\left(s_{2}\right)}-\psi_{\left(s_{1}\right)}$ étant la différence de potentiel imposée aux extrémités de la veine liquide entre les contacts central et périphérique d'un même disque mobile.

Il faut évidemment avoir recours à un calculateur numérique pour résoudre ces équations par itération, en donnant des valeurs différentes à $i_{\mathrm{I}}$ jusqu'à ce que l'éq. (94) soit satisfaite. L'allure des distributions de vitesses obtenues est donnée par les courbes de la figure 7 (courbes I et II $a$ ). La dissymétrie obtenue conduit à deux valeurs différentes $\Delta p_{\mathrm{I}}$ et $\Delta p_{\mathrm{II}}$ de la différence de pression centrifuge entre les extrémités des veines de type I et II respectivement. Pour les distributions représentées sur la figure 7 il résulte que

$$
\Delta p_{\mathrm{I}}>\Delta p_{\mathrm{II}} \text {. }
$$




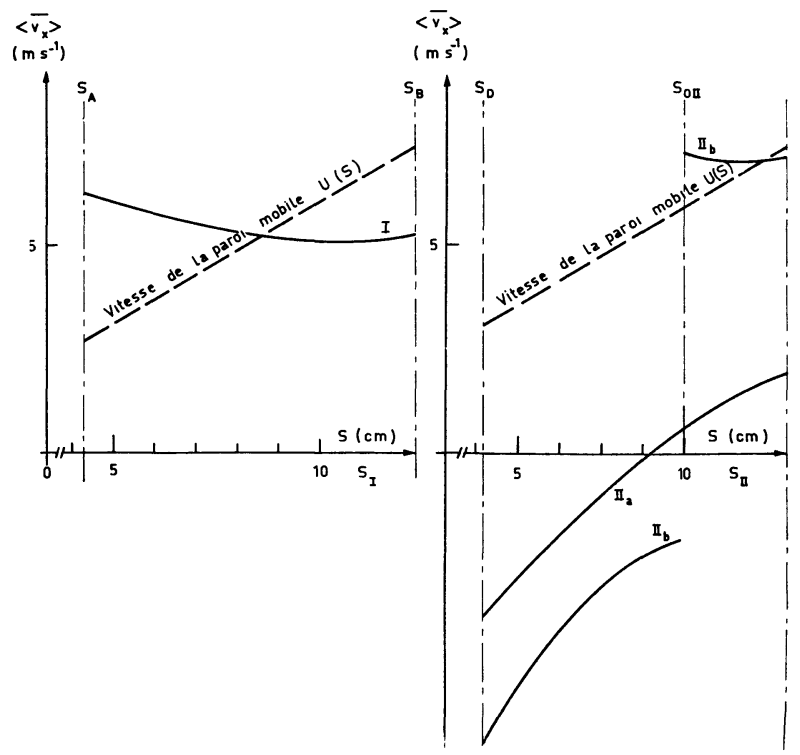

Fig. 7. - Distribution des vitesses moyennes (vitesse circonférentielle) dans les veines liquides I, II ( $a$ et $b$ correspondent à

l'absence ou la présence de dispositif de contre-pression).

Pour une machine à disques multiples où la veine liquide est continue d'un bout à l'autre de l'induit (Fig. 5), cet effet est cumulatif et se traduit par un effort total d'éclatement sur les disques fixes extrêmes. Pour pallier cet inconvénient, nous avons modifié le profil des vitesses (cf. courbe II $b$ de la Fig. 7) en mettant en rotation la veine II de manière telle que l'on ait simultanément dans cette veine, $\Delta \psi_{\mathrm{II}}$ étant la chute ohmique (toujours très faible) créée par le passage du courant d'alimentation de l'induit dans le disque fixe :

$$
\int_{C D} B_{0}\left(s_{\mathrm{II}}\right) \cdot<\bar{v}_{x}>\left(s_{\mathrm{II}}\right) \mathrm{d} s_{\mathrm{II}}=\Delta \psi_{\mathrm{II}} \simeq 0
$$

et

$$
\begin{aligned}
\Delta p_{\mathrm{I}}=\int_{A B} \rho \frac{\left\langle\bar{v}_{x}>^{2}\right.}{s_{\mathrm{I}}} \mathrm{d} s_{\mathrm{I}} & =\Delta p_{\mathrm{II}}= \\
& =\int_{D C} \rho \frac{<\bar{v}_{x}>^{2}}{s_{\mathrm{II}}} \mathrm{d} s_{\mathrm{II}} .
\end{aligned}
$$

Pour réaliser la condition (II), nous avons pratiqué une saignée continue circulaire, d'abscisse curviligne $s_{0_{\text {II }}}=0_{\text {II }} S$ dans le revêtement isolant de la face du disque mobile qui jouxte la veine II, de manière à établir en $S$ un contact électrique entre le disque et la veine [20] et [25].

La fraction de la veine liquide comprise entre $S$ et $C$, continue ainsi à être mise en vitesse dans le sens du disque, alors que la fraction restante comprise entre $S$ et $D$ se met d'elle-même en rotation, en sens inverse, de manière à ce que la condition (I) soit vérifiée. La condition (II) est vérifiée (pour une fréquence de rotation et un courant inducteur donnés) pour une valeur de $s_{0_{\mathrm{II}}}$ déterminée par le calcul.

La connaissance des nouvelles distributions de vitesse (Fig. 7 courbe II $b$ ) permet alors de déterminer les dissipations magnétohydrodynamiques dans la région II.

3. Comparaison des résultats théoriques et expérimentaux. - Les résultats expérimentaux ont été obte-

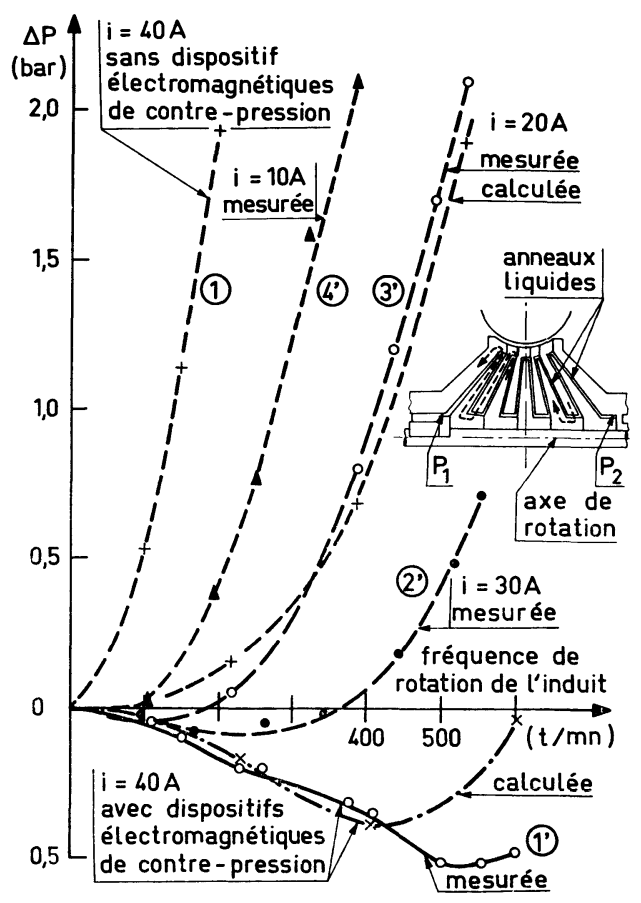

Fig. 8. - Courbe donnant les surpressions totales enregistrées entre les deux extrémités de l'induit, pour différents courants inducteurs $i$, en fonction de la vitesse de rotation des disques. La courbe (1) a été obtenue en l'absence des dispositifs électromagnétiques de contre-pression. Les courbes (1') (2) (3') (4) correspondent aux surpressions mesurées dans le cas de l'utilisation de dispositifs de contre-pression. La machine homopolaire fonctionne alors en moteur à vide (courant d'induit $\simeq 0$ ).

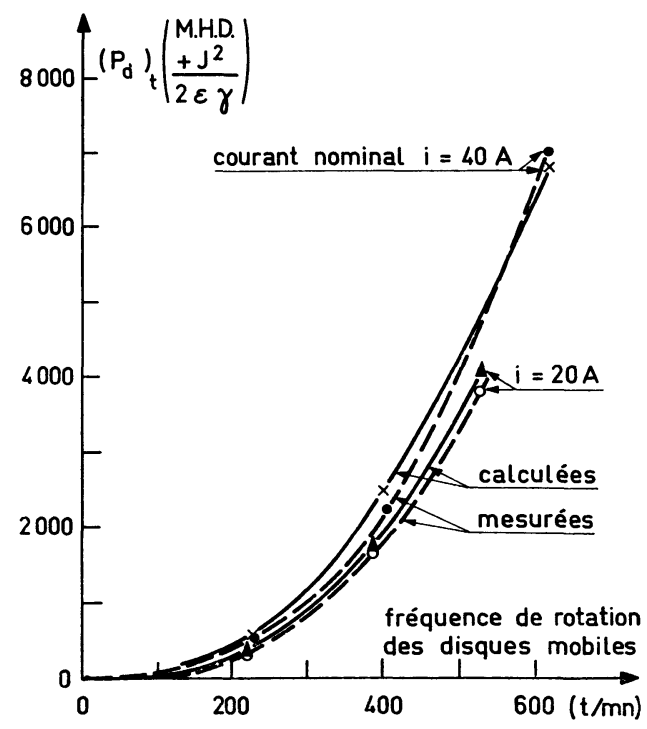

FIG. 9. - Courbe donnant les dissipations totales calculées et mesurées dans l'ensemble des veines liquides de la machine en fonction de la vitesse de rotation des disques et du courant inducteur - $(i=20 \mathrm{~A}$ et $i=40 \mathrm{~A})-$ donc de l'induction extérieure appliquée aux écoulements. 
nus à l'aide d'une maquette probatoire de machine homopolaire possédant les caractéristiques suivantes :

Nombre de disques mobiles d'induit : 5 .

Courant nominal d'induit : $I=15000$ A.

Tension d'alimentation : 4,3 V.

Couple à la vitesse nominale : 815 N.m.

Vitesse nominale : $600 \mathrm{t} \cdot \mathrm{min}^{-1}$.

Courant nominal dans l'inducteur : $i=40 \mathrm{~A}$.

L'induit a un diamètre extérieur de $27 \mathrm{~cm}$ et le rotor une longueur de $22 \mathrm{~cm}$. L'inducteur supraconducteur produit une induction maximale de 2,7 teslas dans le volume utile d'induit à la température ambiante.

Nous avons utilisé les méthodes développées au paragraphe 2.2 pour calculer, au moyen d'un ordinateur B.G.E. 235, les dissipations et les surpressions dans l'ensemble des veines liquides de la machine.
Les courbes des figures 8 et 9 permettent de comparer les résultats théoriques et expérimentaux relatifs :

a) à la différence de pression $\Delta p$ entre les deux extrémités de la machine (Fig. 8),

b) les dissipations totales $\left(P_{\mathrm{d}}\right)_{\mathrm{t}}$ dans l'ensemble des veines liquides.

Ces figures montrent que la méthode de calcul présentée ci-dessus conduit à un bon accord entre les dissipations calculées et mesurées dans les veines latérales (Fig. 9) et à une détermination précise de la vitesse moyenne du fluide $\left\langle\bar{v}_{x}\right\rangle$, les surpressions calculées et mesurées étant très voisines les unes des autres. (Un calcul simple montre que l'erreur commise sur l'évaluation de la surpression $\Delta p$ pour les points $Q$, $Q^{\prime}-600 \mathrm{t} . \mathrm{min}^{-1}, i=40 \mathrm{~A}$ - est $3 \%$.)

\section{Bibliographie}

[1] Rioux, C., Recherche critique d'une forme d'électrotechnique adaptée aux grandes puissances. Thèse, Faculté des Sciences de Paris, 1964.

[2] Rioux, C., Etude et réalisation d'une dynamo unipolaire à régime impulsionnel ne comportant pas de matériaux ferromagnétiques, Ann. Phys. 9 (1964) 729-765.

[3] Chabrerie, J. P., Application des supraconducteurs à l'électrotechnique. Revue Phys. Appl. 4 (1969) 565.

[4] Appleton, A. D., Status of Superconducting machines at I. R. D. Spring 1972. Proceedings of the 1972 Applied Superconductivity Conference, Annapolis, May 1972, I. E. E., Pub. $\mathrm{n}^{\circ}$ 72CHO682-5.

[5] Fox, G. R., HATCH, B. D., Superconducting ship propulsion systems, op. cit. 4.

[6] LANDAU, L. et LIFCHITZ, E., Electrodynamics of continuous Media (Pergamon Press) pp. 214-215.

[7] Murgatroyd, W., Experiments on magneto-hydrodynamic channel flow. Phil. Mag. 441953.

[8] Hartmann, J. and Lazarus, F., Hg. Dynamics II (Experimental investigations on the flox of mercury in a homogeneous magnetic field). Det Kgl. Danske Videnskabernes Selskab. Mathematisk-fysiske Meddelelser XV, 7.

[9] Landau, L. et Lifchitz, F., Fluids Dynamics (Pergamon Press).

[10] HARRIs, L. P., Hydromagnetic channel flows. The Technology Press of the Massachusetts Institue of Technology, and (J. Wiley Inc. New York).

[11] Op. cit. 10, p. 14.

[12] Brouillette, F. C., Lykoudis, P. S., Magneto-fluidmechanic channel flow II. Theory Phys. of fluids 10 (1967) 5995.

[13] Vulis (L. A.) et coll., Resistance in the flow of a liquid metal throug channels in a transverse magnetic field. Magn. Gidrodin. 4 (1968) 19-28.

[14] KIT, L. G., Turbulent velocity fluctuation measurements using a conduction anemometer with a three electrodes probes. Magn. Gidrodin. (1970) 41-46.
[15] Branover, G. G. et coll., Measurements of velocity profiles and fluctuations in a two dimensional channel. Magn. Gidrodin. (1970) 55-58.

[16] Gardner, R. A. et LyKovdis, P. S., Magneto-fluid mechanic pipe flow in a transverse magnetic field. Part. 1 Isothermal flow. J. Fluid Mech. 47 (1971) part 4.

[17] Chabrerie, J. P., Mailfert, A., Robert, J., Filling up sliding electrical contacts for an homopolar engine. Proceedings of the 1968 Holm Seminar on Electric Contact Phenomena, I. I. T. Research Institute, Chicago (USA).

[18] Robertson, J. M. et Johnson, H. F., Turbulence structure in plane Couette flow. Journal of the Engineering Mechanics Division. Proceedings of the American Society of Engineers Dec. 1970, p. 1171-1178.

[19] Chabrerie, J. P., Mailfert, A., Machines unipolaires supraconductrices de faible puissance. Congrès de la Société Française des Electriciens, Nice 1971.

[20] Chabrerie, J. P., Fournet, G., Mailfert, A., Problèmes de magnétohydrodynamique liés à la réalisation d'une machine homopolaire à inducteur supraconducteur et contacts par métaux liquides. C. R. Hebd. Séan. Acad. Sci. 273 (1971) 937-940.

[21] Chabrerie, J. P., Fournet, G., Mailfert, A., Flooded Rotor direct current Acyclic Motor, with Superconducting field winding. Applied Superconductivity Conference, Annapolis, May 1972. I. E. E. Pub. nº 72CHO6825.

[22] Barnes, A. H., US Patent no 2, 588 466, october 24, 1950 and C. J. Mole ; Survey of the state of art of superconducting electrical machinery, nov. 1970. US NAVY Office of Naval Research Contract N 000 14-70 C-0246.

[23] Poulain, J., Contribution à l'étude des machines homopolaires à courant continu. Thèse, Paris, juin 1962.

[24] Robert, J., Contribution à l'étude physico-chimique des amalgames d'indium. Application à l'électrotechnique. Thèse, Paris, juin 1965.

[25] Mailfert, A., Chabrerie, J. P., Perfectionnement aux machines homopolaires à inducteur court. Brevet ANVAR, no 7041767. 